\title{
The PRC-barrel domain of the ribosome maturation protein RimM mediates binding to ribosomal protein S19 in the $30 \mathrm{~S}$ ribosomal subunits
}

\author{
J. MATTIAS LÖVGREN, ${ }^{1}$ GÖRAN O. BYLUND, ${ }^{1,3}$ MANOJ K. SRIVASTAVA, ${ }^{2,4}$ L.A. CARINA LUNDBERG, ${ }^{1,5}$ \\ OLOF P. PERSSON, ${ }^{1}$ GUNNAR WINGSLE, ${ }^{2}$ and P. MIKAEL WIKSTRÖM ${ }^{\mathbf{1}}$ \\ ${ }^{1}$ Department of Molecular Biology, Umeå University, SE-90187 Umeå, Sweden \\ ${ }^{2}$ Swedish University of Agricultural Sciences, Department of Forest Genetics and Plant Physiology, Umeå Plant Science Centre, \\ SE-90183, Umeå, Sweden
}

\begin{abstract}
The RimM protein in Escherichia coli is associated with free $30 \mathrm{~S}$ ribosomal subunits but not with $70 \mathrm{~S}$ ribosomes. A $\Delta$ rimM mutant is defective in $30 \mathrm{~S}$ maturation and accumulates $17 \mathrm{~S}$ rRNA. To study the interaction of RimM with the $30 \mathrm{~S}$ and its involvement in $30 S$ maturation, $\operatorname{RimM}$ amino acid substitution mutants were constructed. A mutant $\operatorname{RimM}(\operatorname{RimM}-\mathrm{YY} \rightarrow \mathrm{AA}$ ), containing alanine substitutions for two adjacent tyrosines within the PRC $\beta$-barrel domain, showed a reduced binding to $30 \mathrm{~S}$ and an accumulation of $17 \mathrm{~S}$ rRNA compared to wild-type RimM. The (RimM-YY $\rightarrow \mathrm{AA})$ and $\Delta$ rimM mutants had significantly lower amounts of polysomes and also reduced levels of $30 \mathrm{~S}$ relative to $50 \mathrm{~S}$ compared to a wild-type strain. A mutation in rpsS, which encodes r-protein S19, suppressed the polysome- and 16S rRNA processing deficiencies of the RimM-YY $\rightarrow$ AA but not that of the $\Delta$ rimM mutant. A mutation in rpsM, which encodes r-protein S13, suppressed the polysome deficiency of both rimM mutants. Suppressor mutations, found in either helices 31 or 33b of 165 rRNA, improved growth of both the RimM-YY $\rightarrow$ AA and $\Delta$ rim M mutants. However, they suppressed the $16 \mathrm{~S}$ rRNA processing deficiency of the RimM-YY $\rightarrow$ AA mutant more efficiently than that of the $\Delta \operatorname{rimM}$ mutant. Helices 31 and 33b are known to interact with S13 and S19, respectively, and S13 is known to interact with S19. A GST-RimM but not a GST-RimM(YY $\rightarrow$ AA) protein bound strongly to S19 in $30 S$. Thus, RimM likely facilitates maturation of the region of the head of $30 S$ that contains S13 and S19 as well as helices 31 and $33 b$.
\end{abstract}

Keywords: RimM; $30 S$ maturation; 16S rRNA processing; S19; S13; helices 31 and 33b

\section{INTRODUCTION}

Up to $40 \%$ of the total energy production in Escherichia coli is consumed by ribosomal biogenesis emphasizing the importance of a coordinated synthesis and assembly of ribosomal components. The $50 \mathrm{~S}$ and $30 \mathrm{~S}$ ribosomal subunits can be reconstituted into fully active ribosomes from the isolated components in vitro. However, nonphysiological

Reprint requests to: P. Mikael Wikström, Department of Molecular Biology, Umeå University, SE-90187 Umeå, Sweden; e-mail: Mikael. Wikstrom@molbiol.umu.se; fax: +46-90-772630.

Present addresses: ${ }^{3}$ Department of Biochemistry and Molecular Biophysics, Washington University School of Medicine, St. Louis, MO 63110, USA; ${ }^{4}$ Department of Biochemistry, JC Bose Institute of Life Sciences, Bundelkhaand University, Jhansi (UP) 284 128, India; ${ }^{5}$ Department of Odontology/Oral Microbiology, Umeå University, SE-90187 Umeå, Sweden.

Article and publication are at http://www.rnajournal.org/cgi/doi/ 10.1261/rna.7720204. conditions like heat-activation steps and different $\mathrm{Mg}^{2+}$ concentrations are required to achieve a reasonable output of mature ribosomal subunits. The maturation of the $50 \mathrm{~S}$ and $30 \mathrm{~S}$ subunits in vivo requires auxiliary proteins that are not part of mature ribosomes. Apart from rRNA processing enzymes and rRNA/r-protein modifying enzymes, a number of other proteins assist in the assembly of the $50 \mathrm{~S}$ and 30 S subunits in E. coli. The DEAD box RNA helicases (Fuller-Pace 1994), SrmB (Nashimoto et al. 1985; Nishi et al. 1988; Charollais et al. 2003), DbpA (Fuller-Pace et al. 1993; Tsu and Uhlenbeck 1998), and CsdA (formerly DeaD) (Toone et al. 1991; Charollais et al. 2004) are assumed to open up RNA structures and allow proper folding of the ribosome. The DnaK chaperone system (Alix and Guérin 1993; Maki et al. 2003), additional heat-shock proteins including GroEL (El Hage et al. 2001), as well as the essential GTPase Era (Nashimoto et al. 1985; Nashimoto 1993), the cold-shock protein RbfA (Dammel and Noller 
1995; Jones and Inouye 1996), and the YrdC protein (Kaczanowska and Rydén-Aulin 2004) are likely involved in ribosome assembly.

The RimM protein is associated with free 30 S subunits but not with $70 \mathrm{~S}$ ribosomes, suggesting an involvement in maturation of the $30 \mathrm{~S}$ subunits or in translation initiation (Bylund et al. 1997). RimM is present in most eubacterial species whose genomes have been sequenced; however, it is not found in any species of the domain Archaea. In addition, RimM-like proteins are reported from at least four eukaryotic species: the malaria parasites Plasmodium falciparum and Plasmodium yoelii, the malaria mosquito Anopheles gambiae, and the chloroplast of the plant Arabidopsis thaliana. An in-frame deletion of $\operatorname{rim} M(\Delta \operatorname{rim} M)$, removing all except the first five and last five codons, decreases the growth rate sevenfold and reduces the translational efficiency at $37^{\circ} \mathrm{C}$ (Bylund et al. 1997). Moreover, the $\Delta$ rimM mutant accumulates 17S rRNA, suggesting a role for RimM in maturation of the 30 S subunits (Bylund et al. 1998). Extragenic suppressor mutations to the $\Delta$ rim $M$ mutation increase the growth rate and translational efficiency of the DrimM mutant (Bylund et al. 1997, 1998, 2001). Some of these mutations alter the carboxy-terminal $16 \mathrm{~S}$ rRNA-binding domain of r-protein S13 (Bylund et al. 1997). The remaining suppressor mutations increase the expression of rbfA (Bylund et al. 1998, 2001). The carboxy-terminal half of RimM was proposed to contain a PRC $\beta$-barrel domain (Anantharaman and Aravind 2002). Previously, a mutant RimM protein (RimM-YY $\rightarrow \mathrm{AA}$ ) containing alanine substitutions for two adjacent tyrosines within the PRC $\beta$-barrel domain showed a reduced binding to the $30 \mathrm{~S}$ ribosomal subunits (Lövgren and Wikström 2001).

In this paper, we show that the RimM-YY $\rightarrow \mathrm{AA}$ mutant accumulated $17 \mathrm{~S}$ rRNA relative to mature $16 \mathrm{~S}$, had lower amounts of $30 \mathrm{~S}$ relative to $50 \mathrm{~S}$ subunits, and had reduced levels of polysomes in comparison to a wild-type strain. Mutations in two adjacent helices (31 and 33b) of 16S rRNA and in the genes for r-proteins S13 and S19 were found to suppress the slow growth of the RimM-YY $\rightarrow$ AA mutant. Furthermore, a GST-RimM wild-type protein bound strongly to S19 in the 30S subunits, whereas a GST$\operatorname{RimM}(\mathrm{YY} \rightarrow \mathrm{AA})$ mutant protein did not. These findings suggest that RimM is important for the maturation of the head region of the $30 \mathrm{~S}$ subunits in which helices 31 and 33b as well as r-proteins S13 and S19 are located.

\section{RESULTS}

\section{Identification of functionally important amino acid residues in $\operatorname{RimM}$}

Functionally important amino acids in RimM were identified by using two different mutagenic approaches. First, to obtain random mutations in rimM, plasmid pMW279, carrying rimM under the control of an IPTG-inducible pro- moter, was mutagenized with hydroxylamine. Derivatives of pMW279 that contained rimM alleles that could not completely complement the slow growth of a $\Delta$ rim $M$ mutant were identified. Two rimM alleles complemented the slow growth at $37^{\circ} \mathrm{C}$ but not at $42^{\circ} \mathrm{C}$ and expressed RimM proteins with the single amino acid substitutions G17E and G27R, respectively. Another rimM allele encoded a RimMG121D protein that showed less complementation than wild-type RimM, when the expression from the IPTG-inducible promoter was limited. The amounts of RimM-G17E and RimM-G27R were 10 -fold higher at both $37^{\circ} \mathrm{C}$ and $42^{\circ} \mathrm{C}$ than the amount of RimM in a wild-type strain (data not shown). Thus, the G17E and G27R substitutions severely affected the function of RimM, and the poor complementation by the two RimM mutant proteins at $42^{\circ} \mathrm{C}$ did not result from a decreased stability at higher temperatures.

Second, to identify conserved amino acids that might be of functional importance, the RimM amino acid sequences from 17 evolutionary distant species were aligned (Fig. 1). Some positions in the amino terminal part of RimM are highly conserved, including G17 and G27, which were identified as important for RimM function in the random mutagenesis screen above. In RimM from E. coli, these glycines are part of the sequences GKMG and GIRG, which are similar to the GXXG motif of the KH domain in RNA-binding proteins (Gibson et al. 1993; Liu et al. 1995). To investigate whether these sequences were important for the binding of RimM to the $30 \mathrm{~S}$ subunits, we replaced the four glycines and the two basic amino acids of the two GXXG motifs with alanines. Strains with these alanine substitutions (G17A, K18A, G20A, G24A, R26A, and G27A) in chromosomally encoded RimM showed growth rates that were similar to that of a wild-type strain at both $37^{\circ} \mathrm{C}$ and $42^{\circ} \mathrm{C}$ (data not shown).

In the proposed PRC $\beta$-barrel domain of RimM, the conserved aromatic amino acids in positions $106-108$, together with the conserved aspartate in position 137 are suggested to bind protein (Anantharaman and Aravind 2002). The two tyrosines in positions 106 and 107 and the aspartate in position 137 were replaced with alanines. The D137A substitution reduced the growth rate at temperatures from $21^{\circ} \mathrm{C}$ to $44^{\circ} \mathrm{C}$, and the effect was more pronounced at the higher temperatures. The growth deficiency was likely due to a decreased stability of the mutant protein, because the RimM levels were more than fivefold lower in the mutant compared to the wild type (data not shown). In contrast, the double substitution Y106A/Y107A reduced the function of RimM, because it lowered the growth rate threefold (Fig. 2) without affecting protein stability (Lövgren and Wikström 2001). The single substitution in position 106 had no effect on the growth rate, whereas that in position 107 slightly reduced the growth rate, especially at $42^{\circ} \mathrm{C}$ (data not shown).

Charged amino acid side chains are likely exposed on the surface of proteins and might, in the case of RimM, interact 


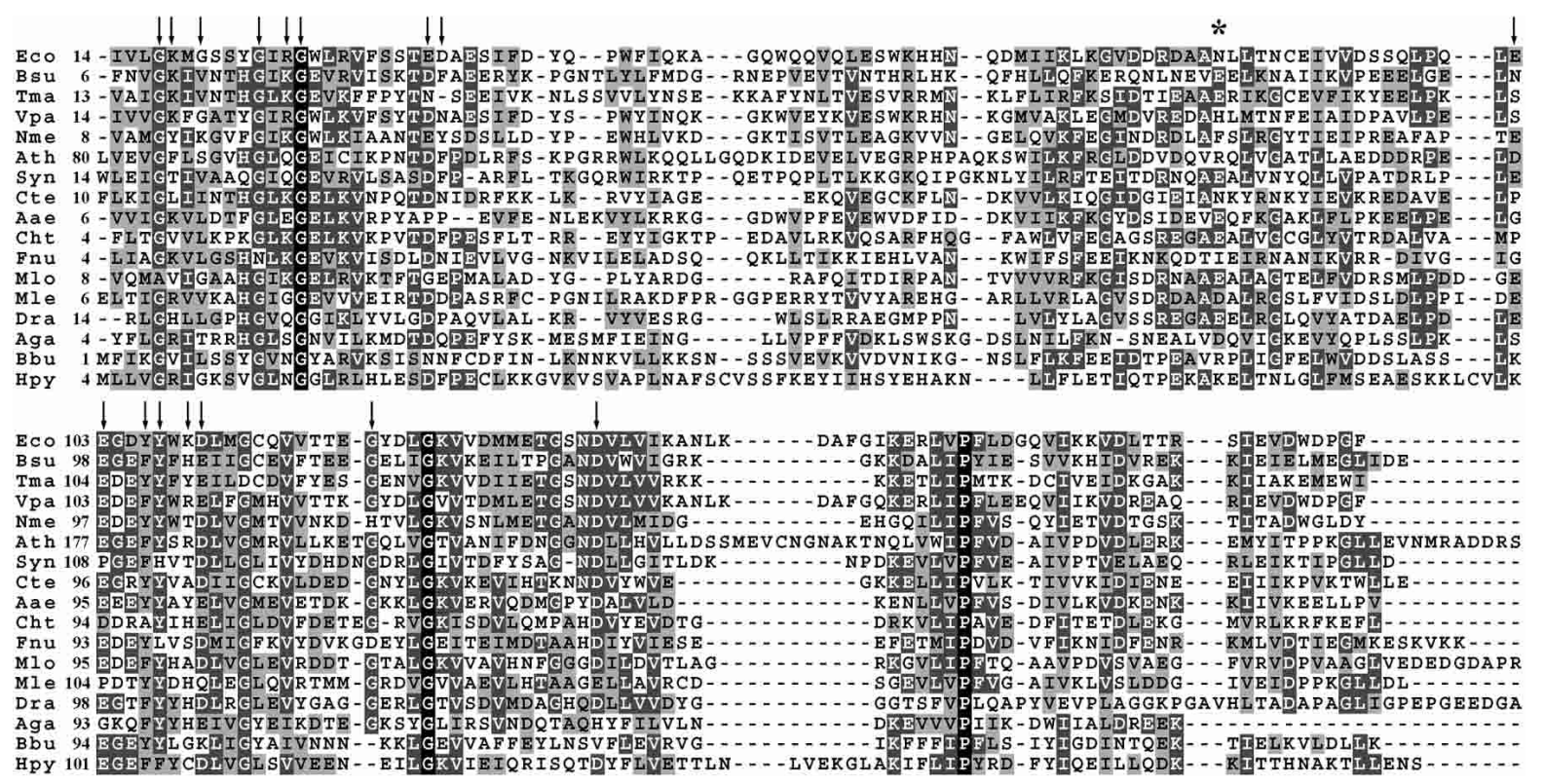

FIGURE 1. Amino acid sequence alignment of RimM from different eubacterial species, the malaria mosquito Anopheles gambiae, and Arabidopsis thaliana. Eco, Escherichia coli; Bsu, Bacillus subtilis; Vpa, Vibrio parahaemolyticus; Nme, Neisseria meningitidis; Ath, Arabidopsis thaliana; Syn, Synechocystis sp. Strain PCC6803; Cte, Clostridium tetani; Aae, Aquifex aeolicus; Cht, Chlorobium tepidum; Fnu, Fusobacterium nucleatum; Mlo, Mesorhizobium loti; Mle, Mycobacterium leprae; Dra, Deinococcus radiodurans; Aga, Anopheles gambiae; Bbu, Borrelia burgdorferi; Hpy, Helicobacter pylori. The numbering to the left of the top row indicates the position in the respective sequence of the first amino acid shown. Amino acid substitutions were isolated for the positions indicated by arrows. The * indicates the position of the N84K substitution suppressing RimM-YY $\rightarrow$ AA.

with the $30 \mathrm{~S}$ subunits. Therefore, charged amino acid residues adjacent to each other in RimM (E36-D37, E102-E103, K109-D110) were replaced by alanines. None of the double substitutions E36A/D37A, E102A/E103A, and K109A/D110A affected the growth rate of the cells on minimal medium plates at $25^{\circ} \mathrm{C}-41.5^{\circ} \mathrm{C}$ or on rich medium plates at $25^{\circ} \mathrm{C}-$ $44^{\circ} \mathrm{C}$ (data not shown).

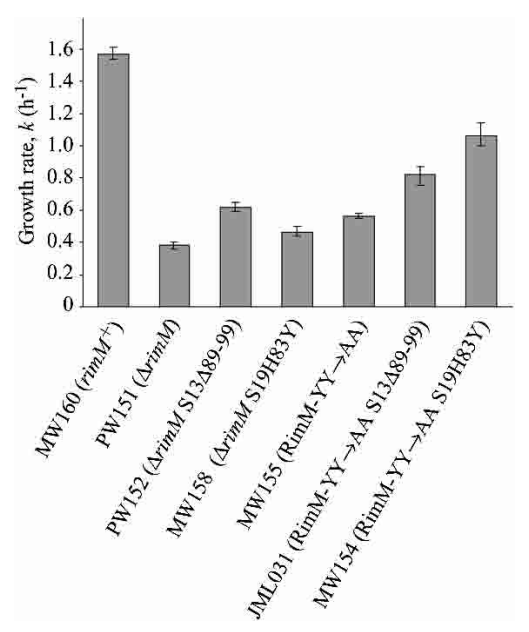

FIGURE 2. The effect of S13 $\Delta 89-99$ and $\mathrm{S} 19 \mathrm{H} 83 \mathrm{Y}$ on the growth rate of the $\Delta$ rim $M$ and RimM-YY $\rightarrow$ AA mutants grown in LB medium at $37^{\circ} \mathrm{C}$. The growth rate was determined in four independent experiments and is expressed as $k(=\ln 2 / g$, where $g$ is the mass doubling time in $h)$. The variation between the experiments is shown as error bars.

\section{Isolation of suppressor mutations that increase the growth rate of the $\mathrm{RimM}-\mathrm{YY} \rightarrow \mathrm{AA}$ mutant}

The RimM-YY $\rightarrow$ AA protein binds less well to the $30 \mathrm{~S}$ subunits than does the wild-type RimM protein (Lövgren and Wikström 2001). The RimM-YY $\rightarrow$ AA mutant was used to select for suppressor mutations that restored binding of RimM-YY $\rightarrow$ AA to the $30 \mathrm{~S}$ subunits resulting in faster growth. One suppressor strain (JML025) contained an N84K substitution in RimM, which increased the growth rate of the RimM-YY $\rightarrow$ AA mutant twofold (the specific growth rate, $k=\ln 2 / g$, where $g$ is the mass doubling time in $\mathrm{h}$, was 1.17 and 0.57 , respectively). The growth rate of the wild-type strain MW100 was 1.61 in the same experiment. To examine whether the N84K substitution also restored the binding of RimM to the 30S subunits, a cell extract of the RimM-YY $\rightarrow$ AA mutant containing the N84K substitution (JML025) was fractionated by sucrose gradient centrifugation and screened for the presence of RimM with an anti-RimM antiserum. No higher amounts of RimM were observed in fractions containing the $30 \mathrm{~S}$ subunits from the RimM-YY $\rightarrow$ AA/N84K suppressor strain compared to those from the RimM-YY $\rightarrow$ AA mutant (data not shown). Thus, the N84K substitution did not restore the binding to the $30 \mathrm{~S}$ subunits, although it improved the function of the $30 \mathrm{~S}$ subunits (see below).

Two of the suppressor strains (JML001, JML026) contained extragenic mutations that were similar to previously isolated mutations in the met $Y$-nusA-infB operon that in- 
crease expression of rbfA (Bylund et al. 2001). Another mutation (spr-8, strain MW145) changed the histidine codon in position 83 of $r p s S$, for r-protein S19, to a tyrosine codon (data not shown). To investigate whether the S19H83Y protein also suppressed the slow growth of a strain lacking RimM, the rpsS mutation was combined with the $\Delta$ rimM mutation. S19H83Y was found to be a weaker suppressor of the $\Delta$ rimM mutant than were mutations that increased expression of $r b f A$, although they all suppressed the slow growth of the RimM-YY $\rightarrow$ AA mutant similarly (data not shown). These results indicated that the H83Y substitution in S19 might have restored a physical interaction between the RimM-YY $\rightarrow$ AA protein and S19. Therefore, a cellular extract of an S19H83Y RimM-YY $\rightarrow$ AA double mutant was fractionated by sucrose gradient centrifugation and screened for the presence of RimM with an anti-RimM antiserum. However, S19H83Y did not increase the amount of the RimM$\mathrm{YY} \rightarrow \mathrm{AA}$ protein associated with the $30 \mathrm{~S}$ subunits (data not shown).

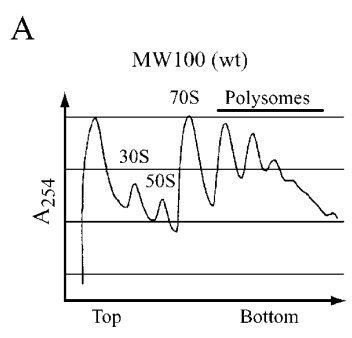

B

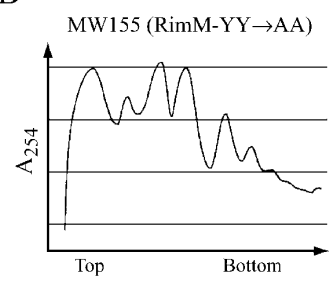

E

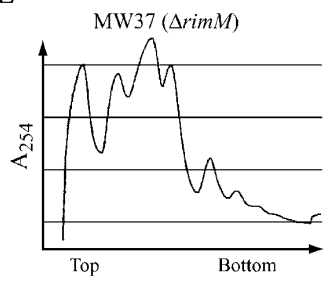

$\mathrm{D}$

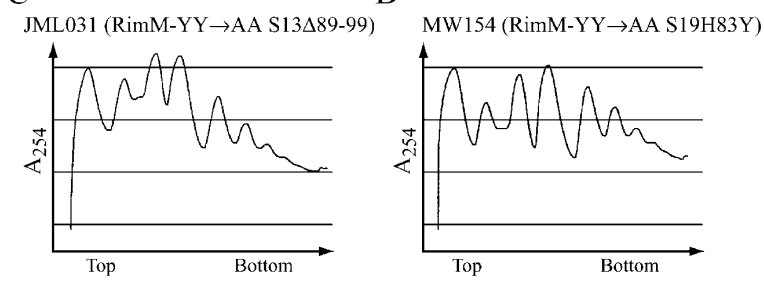

$\mathrm{F}$

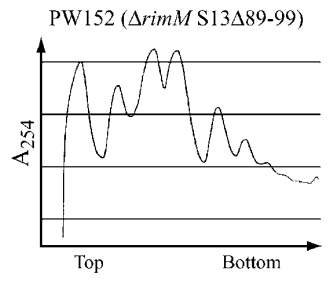

G

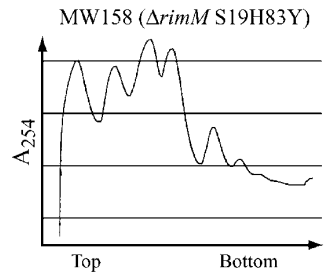

FIGURE 3. The effect of $\mathrm{S} 13 \Delta 89-99$ and $\mathrm{S} 19 \mathrm{H} 83 \mathrm{Y}$ on polysome profiles of the $\Delta$ rimM and $\mathrm{RimM}-\mathrm{YY} \rightarrow \mathrm{AA}$ mutants grown in LB medium at $37^{\circ} \mathrm{C}$. Different ribosome particles are marked above corresponding peaks in $A$.

\section{Effect of alterations in r-proteins S13 and S19 on polysome profiles of the RimM-YY $\rightarrow A A$ and $\Delta$ rimM mutants}

To estimate the translational defects of the RimM-YY $\rightarrow \mathrm{AA}$ and $\Delta$ rim $M$ mutants and to examine the ability of alterations in S13 and S19 to suppress these defects, we analyzed polysome profiles of the different strains by sucrose gradient centrifugation of cellular extracts. The $\Delta$ rim $M$ mutant (MW37) showed a significantly lower amount of polysomes than the wild type (MW100) and, hence, an accumulation of free $30 \mathrm{~S}$ and $50 \mathrm{~S}$ subunits (Fig. 3A,E). The RimM$\mathrm{YY} \rightarrow \mathrm{AA}$ mutant showed a similar translational defect, although it was not as severe as that for the $\Delta \operatorname{rim} M$ mutant (Fig. 3, cf. B and E). Interestingly, both rimM mutants had significantly lower amounts of free $30 \mathrm{~S}$ subunits relative to $50 \mathrm{~S}$ subunits compared to the wild type, indicating that a deficiency in the maturation of the 30 S subunits could explain the low amounts of polysomes. Previously obtained mutations in $r p s M$, which encodes r-protein S13, improved translation in a $\Delta$ rimM mutant (Bylund et al. 1997). The rpsM873 mutation removed amino acids 89 to 99 of S13. The S13 $\Delta 89-99$ protein partially corrected the imbalance in the amount of $30 \mathrm{~S}$ to $50 \mathrm{~S}$ subunits and increased the amount of polysomes of both the RimM-YY $\rightarrow$ AA (Fig. 3C) and the $\Delta$ rimM mutant (Fig. 3F). Also, the S19H83Y protein increased the amount of polysomes of both rimM mutants (Fig. 3D,G). In the RimM-YY $\rightarrow A A$ mutant, the effect of S19H83Y was greater than that of S13 $\Delta 89-99$ (Fig. 3BD), whereas in the $\Delta$ rimM mutant, the effect of S19H83Y was much less than that of S13 $\Delta 89-99$ (Fig. 3E-G). For the two rimM mutants as well as their S13 $\Delta 89-99$ and S19H83Y derivatives, the relative growth rate (Fig. 2) correlated very well with the amount of polysomes detected, demonstrating that the slow growth of rimM mutants is due to poor translation.

\section{Mutations in helices 31 and 33b of 165 rRNA suppress the slow growth of the RimM-YY $\rightarrow A A$ and $\Delta$ rimM mutants}

Conceivably, if RimM interacts with 16S rRNA of the 30S subunits, compensatory mutations in 16S rRNA could be isolated that would restore the interaction between the RimM-YY $\rightarrow$ AA protein and $16 S$ rRNA. However, because E. coli contains seven chromosomal rrn operons (for rRNA), a single mutation may not give any observable suppression. Therefore, we took advantage of an E. coli strain in which all chromosomal $r r n$ operons have been inactivated (Asai et al. 1999a) and the production of ribosomes is dependent on an $r r n$ operon present on plasmid $\mathrm{pHK}-\mathrm{rrnC}^{+}$ 
(Asai et al. 1999b). The rimM mutant gene (rimM120) for $\mathrm{RimM}-\mathrm{YY} \rightarrow \mathrm{AA}$ was introduced into this strain. In this genetic background, RimM-YY $\rightarrow$ AA reduced the growth rate of the cells twofold (Fig. 4A) compared to a threefold reduction in the growth rate of strains containing all chromosomal rrn operons (Fig. 2). Several faster-growing suppressor mutants of the RimM-YY $\rightarrow$ AA mutant (JML068) were isolated, the fastest of which had growth rates that were close to $90 \%$ of the rim $^{+}$strain (Fig. 4A). Twelve suppressor mutations were localized to plasmid $\mathrm{pHK}^{-\mathrm{rnnC}^{+}}$ and identified by DNA sequencing. Ten of the mutations were in four different positions of helix 31, and two were in the identical position of helix 33b of $16 \mathrm{~S}$ rRNA (Fig. 5). To investigate whether the $16 \mathrm{~S}$ rRNA mutations restored the binding of the RimM-YY $\rightarrow \mathrm{AA}$ protein to $30 \mathrm{~S}$ subunits, total cell extracts of three of the suppressor strains (JML075, JML079, and JML098) and of appropriate control strains were subjected to sucrose gradient centrifugation. Fractions were analyzed for the presence of the RimM-YY $\rightarrow$ AA pro-
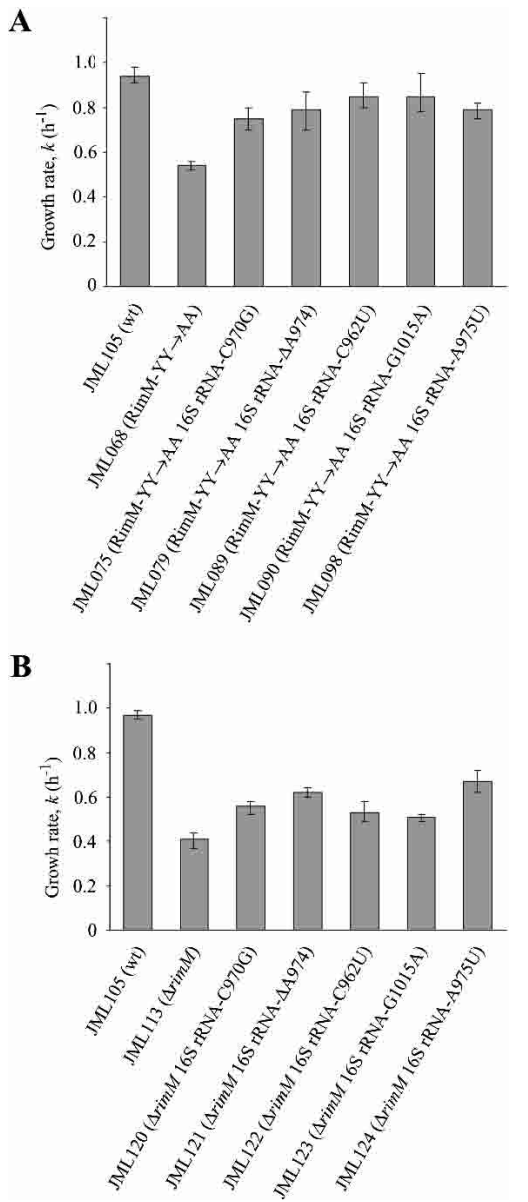

FIGURE 4. The effect of suppressor mutations in helices 31 and $33 \mathrm{~b}$ of $16 \mathrm{~S}$ rRNA on the growth rate of the RimM-YY $\rightarrow$ AA $(A)$ and $\Delta \operatorname{rim} M(B)$ mutants in $\mathrm{LB}$ medium at $37^{\circ} \mathrm{C}$. The growth rate was determined in three independent experiments and is expressed as $k(=\ln 2 / g$, where $g$ is the mass doubling time in $\mathrm{h})$. The variation between the experiments is shown as error bars.

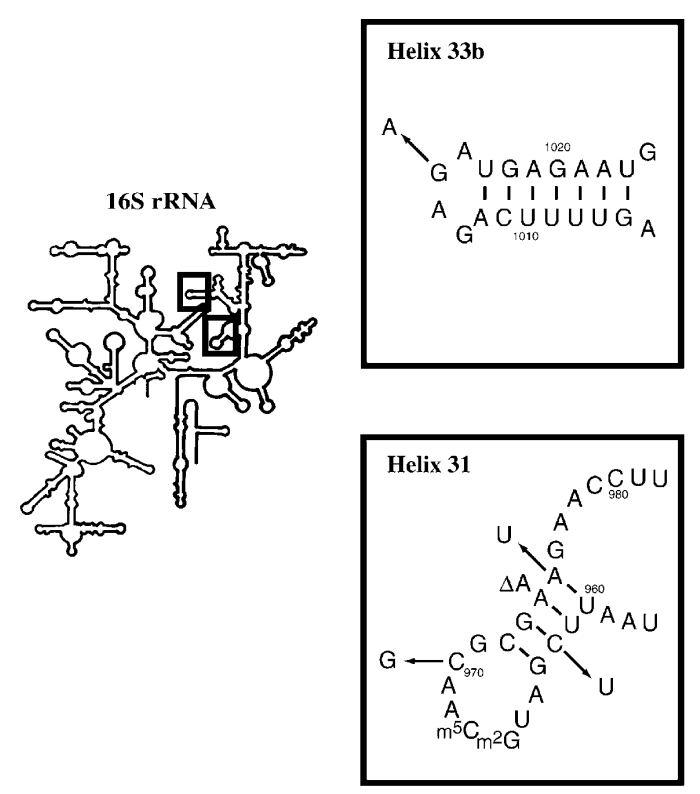

FIGURE 5. Mutations in helices 31 and $33 \mathrm{~b}$ of $16 \mathrm{~S}$ rRNA that suppress the slow growth of the RimM-YY $\rightarrow$ AA and $\Delta$ rim $M$ mutants JML068 and JML114.

tein with an anti-RimM antiserum by Western blotting. The $16 \mathrm{~S}$ rRNA mutations did not restore binding of the RimM$\mathrm{YY} \rightarrow \mathrm{AA}$ protein to the $30 \mathrm{~S}$ subunits (data not shown). To determine whether the $16 \mathrm{~S}$ rRNA mutations could also suppress a $\Delta$ rim $M$ mutant, the suppressor plasmids were substituted for the suppressor-free $r r n$-plasmid pKK1192U in strain JML114 ( $\Delta 7 \mathrm{rrn} \Delta \mathrm{rimM102/pKK1192U}+\mathrm{p} 70)$. All of the mutant plasmids partially suppressed the slow growth of the $\Delta$ rim $M$ mutant, indicating that the suppressor mutations were not allele-specific (Fig. 4B).

\section{$\operatorname{RimM}$ is not directly responsible for the processing of $16 \mathrm{~S}$ rRNA}

The processing of $16 \mathrm{~S}$ rRNA is initiated by RNase III cleavages in a double-stranded region composed of the sequences flanking mature $16 \mathrm{~S}$ rRNA (Young and Steitz 1978). The generated 17S rRNA contains 115 extra nucleotides (nt) at the $5^{\prime}$ end and 33 at the $3^{\prime}$ end compared to mature $16 \mathrm{~S}$ rRNA. In wild-type cells, RNase E and G remove the extra $115 \mathrm{nt}$ at the $5^{\prime}$ end, whereas the removal of the $33 \mathrm{nt}$ at the $3^{\prime}$ end occurs in one step by an unknown RNase and is dependent on the processing by RNase E at the $5^{\prime}$ end (Li et al. 1999). Because a $\Delta$ rim $M$ mutant accumulates 17S rRNA (Bylund et al. 1998), we investigated whether the RimM-YY $\rightarrow$ AA mutant was deficient in processing of $16 \mathrm{~S}$ rRNA. The ability of the RimM-YY $\rightarrow$ AA mutant to convert $17 \mathrm{~S}$ to $16 \mathrm{~S}$ rRNA was determined by primer extension analysis of the $5^{\prime}$-end of $16 \mathrm{~S}$ rRNA. The RimM-YY $\rightarrow$ AA mutant showed five-to-sevenfold increased levels of 17S rRNA compared to the wild type; however, the processing deficiency was not as pronounced as for the 
$\Delta$ rimM mutant (Fig. 6A; Table 1). To examine whether RimM could be the unknown RNase processing the 3 '-end of $17 \mathrm{~S}$ rRNA, total RNA, from a wild-type strain and from the $\Delta$ rimM and RimM-YY $\rightarrow$ AA mutants, was analyzed by Northern blotting with oligonucleotides specific for either the region just upstream of the $3^{\prime}$-end of mature 16S rRNA or the extra nucleotides present in 17S rRNA. The two rimM mutants showed only a partial accumulation of unprocessed 3 ' ends (data not shown), mimicking the results for the processing of the $5^{\prime}$ end in the same mutants. Thus, RimM cannot be the unknown RNase that processes the 3' end of 16S rRNA, and the observed partial processing deficiency was probably an indirect effect of poor processing by RNase $\mathrm{E}$ at the $5^{\prime}$ end.

\section{The effect of the suppressor mutations on the processing of $16 \mathrm{~S}$ rRNA}

The 16S rRNA processing deficiency of rimM mutants is likely the result of a defect in a step in the maturation of the $30 \mathrm{~S}$ subunits that precedes the final processing of $17 \mathrm{~S}$ into $16 \mathrm{~S}$ rRNA (Bylund et al. 1998). To examine whether mutations suppressing the two rim $M$ mutations improved the processing of $16 \mathrm{~S}$ rRNA, we determined the processing ef- ficiency of different suppressor strains by primer extension analysis of the $5^{\prime}$-end of $16 \mathrm{~S}$ rRNA. Interestingly, S19H83Y suppressed partially the processing deficiency of the RimM$\mathrm{YY} \rightarrow \mathrm{AA}$ mutant but not that of the $\Delta$ rimM mutant (Fig. $6 \mathrm{~A}$; Table 1). S13 $\Delta 89-99$, on the other hand, did not suppress the processing deficiency of either of the rimM mutants (Fig. 6A; Table 1). To our surprise, the N84K substitution in the RimM-YY $\rightarrow \mathrm{AA}$ protein improved the processing efficiency threefold (Table 1), although it did not improve the binding of RimM-YY $\rightarrow$ AA to the 30 S subunits (see above). The five different suppressor mutations in the plasmid-carried 16S rRNA gene improved the processing of $16 \mathrm{~S}$ rRNA in the RimM-YY $\rightarrow$ AA mutant 1.5-to-fourfold, whereas they only improved the processing 1.6-fold or less in the $\Delta$ rimM mutant (Fig. 6B; Table 2). Note that the mutation with the least effect (A975U) in the RimM-YY $\rightarrow$ AA mutant (JML098) was the one that had the greatest effect in the $\Delta$ rimM mutant (JML124).

\section{The tyrosines of the PRC-barrel domain of RimM bind specifically to r-protein S19 in the $30 \mathrm{~S}$ subunits}

RimM is found associated with free $30 \mathrm{~S}$ subunits but not with $70 \mathrm{~S}$ ribosomes (Bylund et al. 1997). To identify the
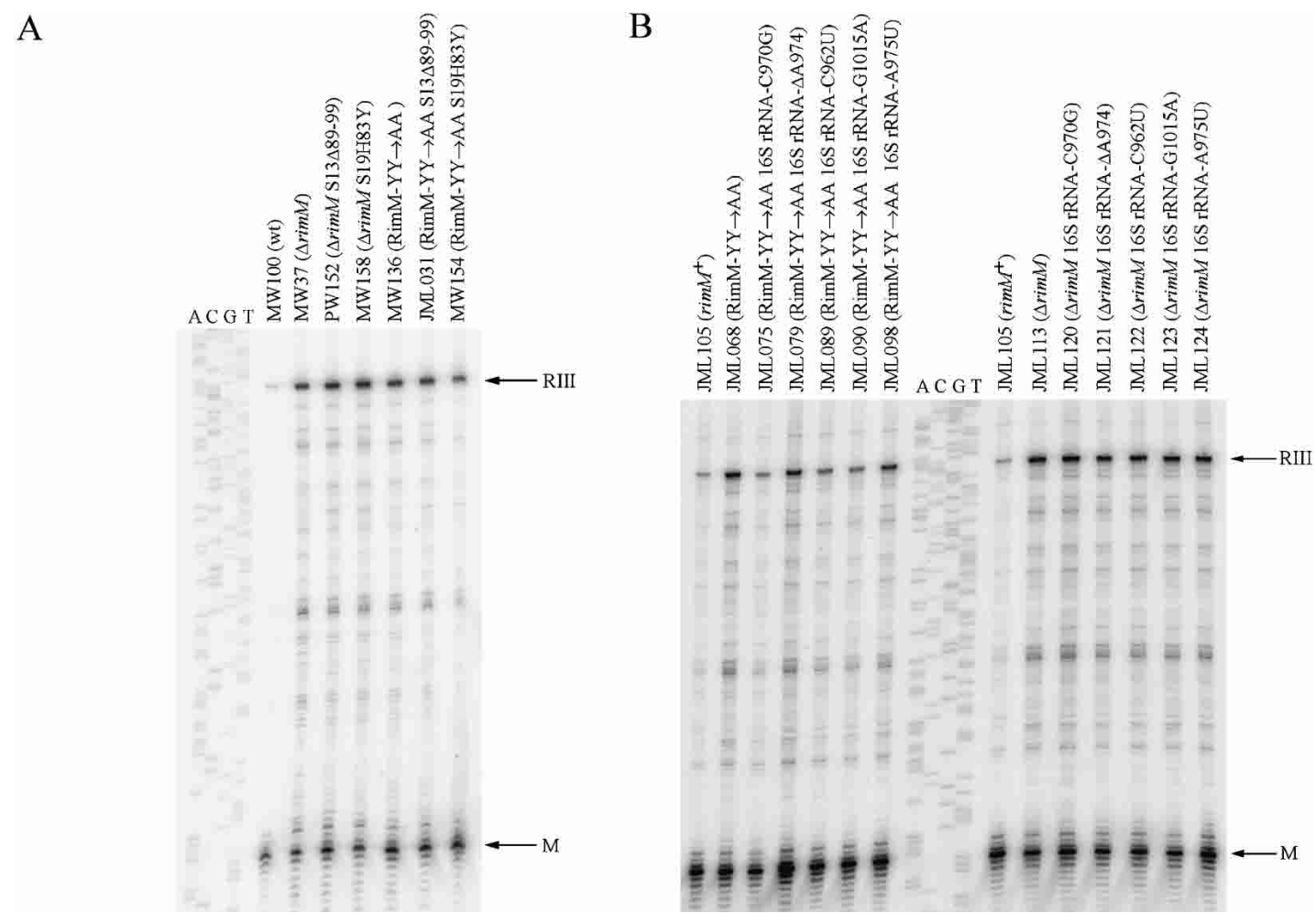

FIGURE 6. The effect of different suppressor mutations on the $16 \mathrm{~S}$ rRNA processing deficiency of rimM mutants. The $5^{\prime}$-end of $16 \mathrm{~S}$ rRNA was determined by primer extension analysis. RIII indicates a primer extension product of $179 \mathrm{nt}$ corresponding to $17 \mathrm{~S}$ rRNA processed at the RNase III site $115 \mathrm{nt}$ upstream of the $5^{\prime}$-end of mature $16 \mathrm{~S}$ rRNA. M indicates a product of $64 \mathrm{nt}$ corresponding to the $5^{\prime}$-end of mature $16 \mathrm{~S}$ rRNA. The sequencing ladder shown is of $r r \mathrm{C}$ in plasmid $\mathrm{pHK}-\mathrm{rrnC}^{+}$and was obtained by using the same primer as for the primer extension reactions. $(A)$ The effect of S13 $\Delta 89-99$ and S19H83Y on the 16S rRNA processing in $\Delta$ rimM and RimM-YY $\rightarrow$ AA mutants. (B) The effect of $16 S$ rRNA mutations on $16 \mathrm{~S}$ rRNA processing in $\Delta$ rim $M$ and RimM-YY $\rightarrow$ AA mutants. 
TABLE 1. The effect of S19H83Y, S13 $889-99$, or RimM-N84K on the processing of $16 \mathrm{~S}$

\begin{tabular}{|c|c|c|c|c|c|}
\hline \multirow[b]{2}{*}{ Strain } & \multirow[b]{2}{*}{ Relevant traits } & \multicolumn{4}{|c|}{ Accumulation of $17 \mathrm{~S} r R N A^{a}$} \\
\hline & & Exp. I & Exp. II & Exp. III & Exp. IV \\
\hline MW100 & wt & 0.074 & 0.029 & 0.051 & 0.061 \\
\hline MW136 & $\operatorname{RimM}-\mathrm{YY} \rightarrow \mathrm{AA}$ & 0.39 & 0.18 & 0.38 & 0.39 \\
\hline MW154 & RimM-YY $\rightarrow$ AA S19H83Y & 0.26 & 0.087 & n.d. & n.d. \\
\hline JML031 & $\operatorname{RimM} M-Y Y \rightarrow$ AA S13 $\Delta 89-99$ & 0.43 & 0.21 & n.d. & n.d. \\
\hline JML025 & $\operatorname{RimM}-\mathrm{YY} \rightarrow \mathrm{AA} / \mathrm{N} 84 \mathrm{~K}$ & n.d. & n.d. & 0.12 & 0.14 \\
\hline MW37 & $\operatorname{\Delta rimM}$ & 0.54 & 0.33 & n.d. & n.d. \\
\hline MW158 & $\Delta \operatorname{rimM} \mathrm{S} 19 \mathrm{H} 83 \mathrm{Y}$ & 0.64 & 0.32 & n.d. & n.d. \\
\hline PW152 & $\Delta \operatorname{rimM}$ S13 $\Delta 89-99$ & 0.52 & 0.34 & n.d. & n.d. \\
\hline
\end{tabular}

aThe amount of 17S rRNA to the total amount of $16 \mathrm{~S}$ rRNA [RIII / (M + RIII)] was calculated from the amount of radioactivity in the primer extension products obtained in experiments like that shown in Figure 6A. rRNA in rimM mutants

pared and analyzed at room temperature $\left(21^{\circ} \mathrm{C}\right)$, most $30 \mathrm{~S}$ subunit r-proteins were released from the column at washes with up to $0.6 \mathrm{M} \mathrm{NaCl}$ (Fig. 8A). All 30 S structural proteins except S6, S12, S15, S17, S18, S19, S20, and S21 were identified when total protein in the $0.6 \mathrm{M} \mathrm{NaCl}$ wash fraction was analyzed by ESI-MS/MS (data not shown). The most intense protein bands $(\leq 30 \mathrm{kDa}$ in Fig. 8A) were identified as r-proteins S2, S3, S4, S5, S7, S9, S10, S11, S13, and S17 by MALDI mass spectrometry. Moreover, 16S rRNA was present in the $0.6 \mathrm{M} \mathrm{NaCl}$ wash fraction as shown by primer extension analysis (data not shown), suggesting that intact $30 \mathrm{~S}$ subunits were initially bound to the GST- binding site for RimM in the $30 \mathrm{~S}$ subunits, a hybrid protein between glutathione S-transferase (GST) and RimM was used to pull out proteins interacting with RimM. The GSTRimM protein expressed from plasmid pJML005 complemented the slow growth of a $\Delta$ rimM mutant (GOB191), suggesting that the RimM part of the hybrid protein was functional (data not shown). The GST-RimM protein from total cell extracts of strain GOB191/pJML005 was bound to a glutathione column, which was then washed with increasing concentrations of $\mathrm{NaCl}$. Finally, the GST-RimM protein plus proteins bound to RimM were eluted from the column. When extracts were prepared and analyzed at $4^{\circ} \mathrm{C}$, several proteins smaller than $30 \mathrm{kDa}$ in size were found in the stringent wash fractions and in the eluate (Fig. 7A). The eluate contained all small subunit r-proteins except S3, S14, $\mathrm{S} 16$, and $\mathrm{S} 21$ as determined by electro-spray ionization tandem mass spectrometry (ESI-MS/MS) (data not shown). In addition, r-protein L1 and some proteins not involved in translation were found in the eluate. Further, the eluate was shown by primer extension analysis to contain 16S rRNA (Fig. 7C), indicating that the GSTRimM protein bound to $30 \mathrm{~S}$ subunits and not to free r-proteins. In contrast, when extracts of a strain expressing a $\mathrm{GST}-\operatorname{RimM}(\mathrm{YY} \rightarrow \mathrm{AA})$ mutant protein were analyzed, no r-proteins were present in the eluate or in the stringent wash fractions (Fig. 7B). Thus, these results are consistent with the previous findings that the two conserved tyrosines are important for the binding of RimM to the $30 \mathrm{~S}$ subunits (Lövgren and Wikström 2001).

When extracts that contained the GST-RimM wild-type protein were prein rimM mutants
RimM protein not only at $4^{\circ} \mathrm{C}$ but also at room temperature. No r-proteins were found in the $0.6 \mathrm{M} \mathrm{NaCl}$ wash fraction when an extract of the strain expressing the GST$\operatorname{RimM}(\mathrm{YY} \rightarrow \mathrm{AA})$ mutant protein was analyzed by ESI-MS/ MS (Fig. 8B; data not shown). Interestingly, r-protein S19, as identified by MALDI mass spectrometry (data not shown), copurified with the GST-RimM wild-type protein but not with the GST-RimM(YY $\rightarrow$ AA) mutant protein (Fig. 8, cf. A and B). These findings demonstrate that the tyrosines present in positions 106 and 107 of wild-type RimM but absent in the RimM-YY $\rightarrow$ AA protein are crucial for the binding of RimM to S19 in the 30S subunits. Note also that S19 was not present among those r-proteins that were identified in the $0.6 \mathrm{M} \mathrm{NaCl}$ wash fraction when the GST-RimM wild-type protein was purified, suggesting that S19 bound strongly to the GST-RimM protein. Thus, the
TABLE 2. The effect of suppressor mutations in $16 \mathrm{~S}$ rRNA on the processing of $16 \mathrm{~S}$ rRNA

\begin{tabular}{|c|c|c|c|c|c|}
\hline \multirow[b]{2}{*}{ Strain } & \multirow[b]{2}{*}{ Relevant traits } & \multicolumn{4}{|c|}{ Accumulation of $17 \mathrm{~S} r \mathrm{RNA}^{\mathrm{a}}$} \\
\hline & & Exp. I & Exp. II & Exp. III & Exp. IV \\
\hline JML105 & RimM-wt 16S rRNA-wt & 0.066 & 0.016 & 0.039 & 0.075 \\
\hline JML068 & RimM-YY $\rightarrow$ AA 16S rRNA-wt & 0.32 & 0.14 & 0.23 & n.d. \\
\hline JML075 & RimM-YY $\rightarrow$ AA 16S rRNA-C970G & 0.12 & 0.047 & 0.058 & n.d. \\
\hline JML079 & $\operatorname{RimM-YY\rightarrow AA~16S~rRNA-~} \Delta$ A974 & 0.18 & 0.051 & 0.052 & n.d. \\
\hline JML089 & RimM-YY $\rightarrow$ AA 16S rRNA-C962U & 0.13 & 0.046 & 0.092 & n.d. \\
\hline JML090 & RimM-YY $\rightarrow$ AA 16S rRNA-G1015A & 0.12 & 0.037 & 0.098 & n.d. \\
\hline JML098 & $\operatorname{RimM-YY\rightarrow AA~16S~rRNA-A975U~}$ & 0.23 & 0.075 & 0.15 & n.d. \\
\hline JML113 & $\Delta \operatorname{rim} M 16 \mathrm{~S}$ rRNA-wt & 0.46 & n.d. & n.d. & 0.54 \\
\hline JML120 & arimM 16S rRNA-C970G & 0.40 & n.d. & n.d. & 0.45 \\
\hline JML121 & $\Delta \operatorname{rimM} 16 \mathrm{~S}$ rRNA- $\Delta \mathrm{A} 974$ & 0.38 & n.d. & n.d. & 0.32 \\
\hline JML122 & $\Delta \operatorname{rimM} 16 \mathrm{~S}$ rRNA-C962U & 0.42 & n.d. & n.d. & 0.36 \\
\hline JML123 & $\Delta$ rimM 16S rRNA-G1015A & 0.45 & n.d. & n.d. & 0.40 \\
\hline JML124 & srimM 16S rRNA-A975U & 0.30 & n.d. & n.d. & 0.32 \\
\hline
\end{tabular}

aThe amount of 17S rRNA to the total amount of 16S rRNA [RIII / (M + RIII)] was calculated from the amount of radioactivity in the primer extension products obtained in experiments like that shown in Figure 6B. 
A

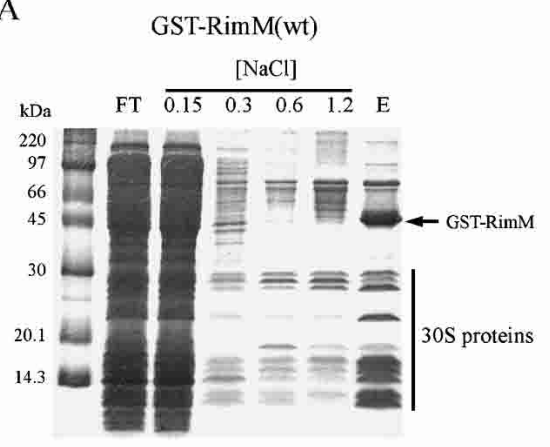

B

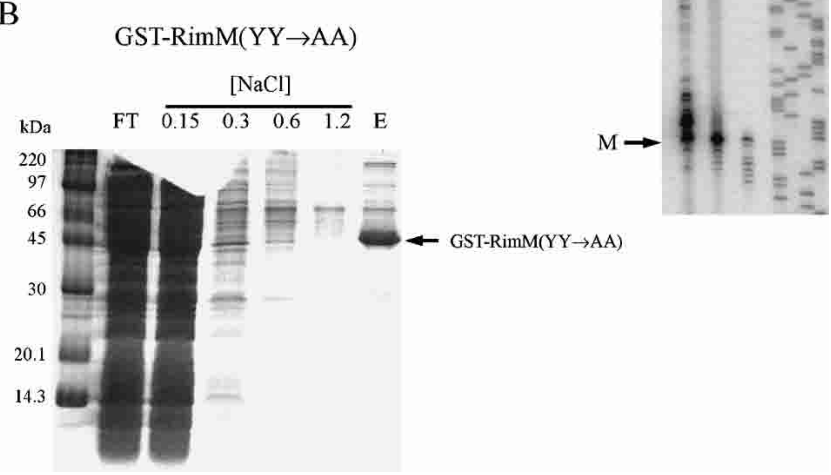

FIGURE 7. Analysis of cellular components copurifying with GSTRimM hybrid proteins at $4^{\circ} \mathrm{C}$. (A) A cell extract of strain GOB191/ pJML005 ( $\Delta$ rimM/GST-RimM) was prepared by freeze-thawing, and the GST-RimM hybrid protein in the extract was adsorbed to Glutathione Sepharose 4B, washed at the indicated molar concentrations of $\mathrm{NaCl}$ and finally eluted by reduced glutathione, all at $4^{\circ} \mathrm{C}$. FT, flowthrough; E, eluate. (B) As in A, but the strain used was GOB191/ pJML051 $[\Delta \operatorname{rimM} / \mathrm{GST}$-RimM(YY $\rightarrow \mathrm{AA})]$. (C) Primer extension analysis of $16 \mathrm{~S}$ rRNA prepared from the flow-through and eluate from $A$, and from $30 \mathrm{~S}$ subunits obtained after sucrose gradient centrifugation of a total cell extract of the wild-type strain MW100.

interaction between RimM and S19 at room temperature seems to be stronger than that between S19 and other components of the $30 \mathrm{~S}$ subunits, at least at higher $\mathrm{NaCl}$ concentrations.

The $30 \mathrm{~S}$ subunits bound to the GST-RimM wild-type protein both at $4^{\circ} \mathrm{C}$ and at room temperature contained mature 16S rRNA and almost no 17S rRNA. This was unexpected, because a $\Delta$ rimM mutant accumulates $17 \mathrm{~S}$ rRNA and therefore RimM has been suggested to bind 30S subunits that contain $17 \mathrm{~S}$ rRNA rather than fully processed 16S rRNA.

To investigate whether S19H83Y, which improved the processing of $16 \mathrm{~S}$ rRNA of the RimM-YY $\rightarrow$ AA mutant but not that of the $\triangle$ rim $M$ mutant, could restore the binding of the RimM-YY $\rightarrow$ AA protein to the $30 \mathrm{~S}$ subunits, the GST$\operatorname{RimM}$ and GST-RimM(YY $\rightarrow$ AA) proteins were purified from strains expressing the S19H83Y protein. The S19H83Y protein copurified with the GST-RimM protein only (Fig. $8 \mathrm{C})$ and not with the GST-RimM(YY $\rightarrow$ AA) protein (Fig. $8 \mathrm{D})$ when extracts were prepared and analyzed at room temperature. Further, no larger amounts of r-proteins were found in the wash fractions of the S19H83Y GST$\operatorname{RimM}(\mathrm{YY} \rightarrow \mathrm{AA})$ strain than in those of the S19-wt GST$\operatorname{RimM}(\mathrm{YY} \rightarrow \mathrm{AA})$ strain. Thus, S19H83Y did not seem to restore the binding of the RimM-YY $\rightarrow \mathrm{AA}$ protein to the 30 S subunits.

\section{DISCUSSION}

\section{Identification of functionally important positions in RimM}

The introduction of charged amino acids in two of the most conserved positions of RimM (G17E and G27R) dramatically reduced the stability of the protein (data not shown), but also reduced its function at high temperature without a further decrease in stability, implying a structural role of the conserved glycines. More subtle substitutions in the same positions (G17A and G27A) had no significant effects on the function of RimM. Because alanine for glycine substitutions are believed to affect the function of a protein mainly when glycines are important for the flexibility of a polypeptide chain, our results suggest other structural roles for these glycines in RimM. The double substitution Y106A/ Y107A reduced the affinity of RimM for the 30S subunits, implying that the two tyrosines are important for the binding of RimM to the 30S subunits (this paper; Lövgren and Wikström 2001). These tyrosines together with the highly conserved aspartate in position 137 of a proposed PRC $\beta$-barrel domain are suggested to line a cleft into which a peptide of an interacting protein could be accommodated (Anantharaman and Aravind 2002). The D137A substitution severely reduced the stability of RimM, which precluded the possibility to assess whether or not this position in RimM interacts with the $30 \mathrm{~S}$ subunits. However, the effect on the stability suggests an important role of this aspartate for the structure and stability of the PRC domain. Another substitution (G121D) in the PRC domain also affected the structure and/or function of RimM, as plasmidencoded RimM variants with this substitution showed a reduced ability to complement chromosomal deletions of rimM.

\section{Identification of the target region for $\operatorname{RimM}$ in the $30 \mathrm{~S}$ subunits}

Alterations in r-proteins S13 and S19 as well as mutations in helices 31 and $33 \mathrm{~b}$ of $16 \mathrm{~S}$ rRNA suppress the slow growth of a RimM-YY $\rightarrow$ AA mutant and to different degrees that of a $\Delta$ rimM mutant. These alterations are all located in a distinct region of the head of the 30 S subunit (Fig. 9) in which helix 33 b interacts with S19, helix 31 contacts S13, and S13 and S19 interact with each other (Wimberly et al. 2000). In addition, wild-type RimM, in contrast to the RimM-YY $\rightarrow$ AA 
A

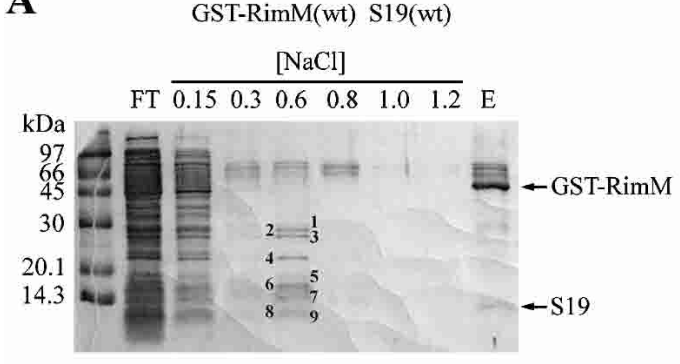

C

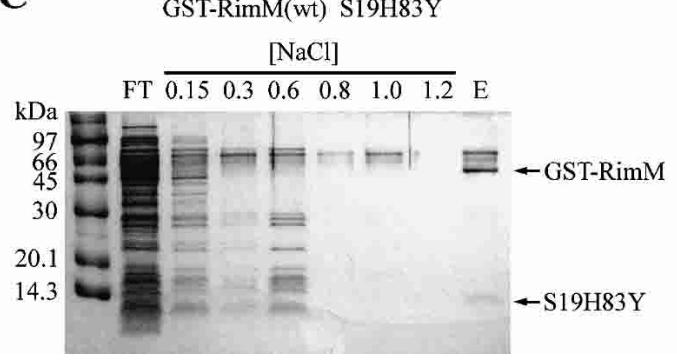

B

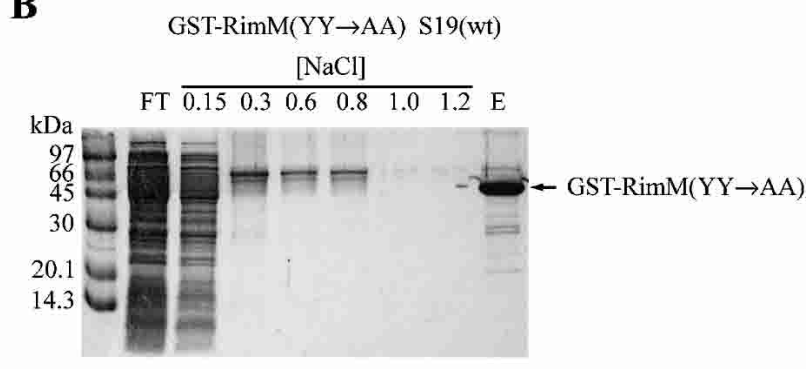

D

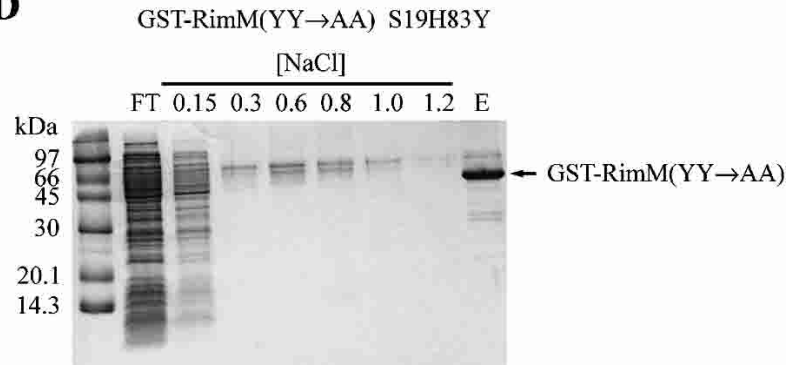

FIGURE 8. Analysis of cellular components copurifying with GST-RimM hybrid proteins at $21^{\circ} \mathrm{C}$. Cellular extracts were prepared by sonication and the GST-RimM hybrid proteins were purified as described in the Figure 7 legend. Similar results were obtained when extracts were prepared by freeze-thawing. (A) Strain GOB191/pJML005 ( $\Delta$ rimM S19-wt/GST-RimM). The proteins indicated in the lane with the $0.6 \mathrm{M}$ NaCl wash fraction were identified as $30 \mathrm{~S}$ subunit r-proteins by MALDI mass spectrometry: $1, \mathrm{~S} 2 ; 2, \mathrm{~S} 3 ; 3, \mathrm{~S} 4 ; 4, \mathrm{~S} 7 ; 5, \mathrm{~S} 5 ; 6, \mathrm{~S} 9 ; 7, \mathrm{~S} 11$ and S13; 8, S10; and 9, S17. In addition, one weak band above that containing S2 was identified as r-protein L2. (B) Strain GOB191/pJML051 [ $\Delta$ rimM S19-wt/GST-

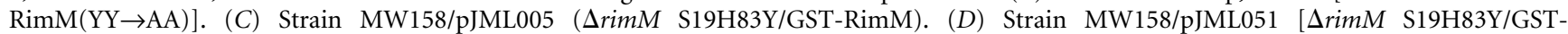
$\operatorname{RimM}(\mathrm{YY} \rightarrow \mathrm{AA})]$.

protein, bound strongly to S19 in the $30 \mathrm{~S}$ subunits, suggesting that the tyrosines in positions 106-107 of RimM mediate the binding to S19. One possible role for RimM in ribosome maturation might be to facilitate the interaction of S19 with helix 33b, and perhaps also the interaction between S13 and helix 31. In the maturation of the 30S subunits, the $5^{\prime}$-end processing of $16 \mathrm{~S}$ rRNA has been suggested to be one of the last steps, which would also activate the 30 S subunits (see Nierhaus 1991). Conceivably, a defect in any step in the $30 \mathrm{~S}$ maturation process that precedes the processing of the $5^{\prime}$-end of $16 \mathrm{~S} \mathrm{rRNA}$ might result in a $16 \mathrm{~S}$ rRNA processing deficiency and also a translational deficiency. Thus, the $16 \mathrm{~S}$ rRNA processing deficiency observed for the $\Delta$ rimM and RimM-YY $\rightarrow \mathrm{AA}$ mutants (this paper; Bylund et al. 1998) could stem from poor or inappropriate interactions in the S13-S19 region of the 30S subunits. Interestingly, S19 plays a key role in r-protein-dependent conformational rearrangements of $16 \mathrm{~S}$ rRNA during $30 \mathrm{~S}$ maturation in vitro (Holmes and Culver 2004).

\section{$\operatorname{RimM}-\mathrm{YY} \rightarrow \mathrm{AA}$-dependent and $\operatorname{RimM}-\mathrm{YY} \rightarrow \mathrm{AA}$-independent suppression}

The S19H83Y protein partially restored the ability of the RimM-YY $\rightarrow$ AA mutant to process $16 \mathrm{~S}$ rRNA and to form polysomes. However, S19H83Y had less effect on the growth and polysome deficiencies and no effect on the $16 \mathrm{~S}$
rRNA processing deficiency of the $\Delta$ rim $M$ mutant. Thus, to mediate efficient suppression, $\mathrm{S} 19 \mathrm{H} 83 \mathrm{Y}$ is dependent on the presence of the RimM-YY $\rightarrow$ AA protein, especially for the

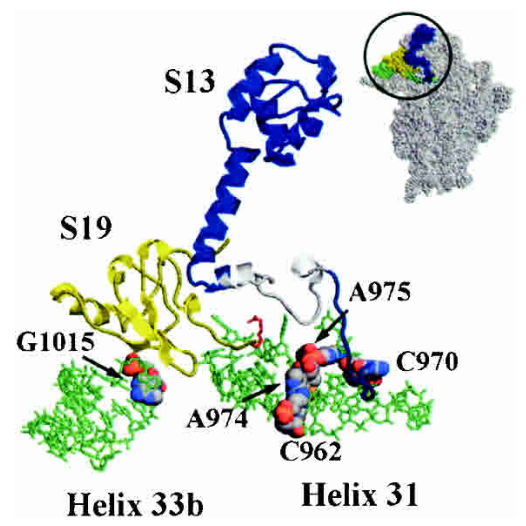

FIGURE 9. Alterations in the E. coli 30 S subunit (indicated in the model for the $30 \mathrm{~S}$ subunit of Thermus thermophilus) that suppress rimM mutations. The structure of the $30 \mathrm{~S}$ subunit is from Wimberly et al. (2000) and was retrieved from the Protein Data Bank (PDB no. 1FJF). Only part of the structure is shown. Helices 31 and $33 \mathrm{~b}$ of $16 \mathrm{~S}$ rRNA are presented as stick models in green except for the positions with suppressor mutations, which are in space-fill models. R-protein $\mathrm{S} 13$ is shown in a blue ribbon model except for a region in gray, which contains the alterations that suppress rimM mutations. S19 is shown in a yellow ribbon model with the most carboxy terminal amino acid, R81, of the determined structure, colored red. Thus, the alteration, $\mathrm{H} 83 \mathrm{Y}$, in $\mathrm{S} 19$ that suppressed RimM-YY $\rightarrow \mathrm{AA}$ is in a position just outside of the determined structure. 
processing of $16 \mathrm{~S}$ rRNA. Four of the five different mutations in helices 31 and 33b of $16 \mathrm{~S}$ rRNA were more efficient suppressors of the 16S rRNA processing deficiency of the strain containing the RimM-YY $\rightarrow$ AA protein compared to that of a mutant lacking RimM, although the 16S rRNA mutations had similar effects on the growth rate of the two rimM mutants. The fifth 16S rRNA mutation (A975U) suppressed the processing deficiency similarly in the two rimM mutants; however, it was the weakest of the five suppressor mutations in the RimM-YY $\rightarrow$ AA mutant. Interestingly, the effect of the 16S rRNA suppressor mutations on the growth rate did not correlate with their effect on the processing of $16 \mathrm{~S}$ rRNA. Further, S13 $\Delta 89-99$ increased the growth rate of and amount of polysomes in the $\Delta r i m M$ and RimM-YY $\rightarrow$ AA mutants, although it did not correct the $16 \mathrm{~S}$ rRNA processing deficiency. Taken together, these findings suggest that for the suppression of the translational defects of the rimM mutants, there are two different mechanisms. One mechanism improves the function of $30 \mathrm{~S}$ subunits containing fully processed 16S rRNA. This is corroborated by earlier results showing that mature ribosomes in a strain lacking RimM are not as efficient as wild-type ribosomes in translating the lacZ mRNA (Bylund et al. 1997). A second mechanism improves the maturation of the $30 \mathrm{~S}$ subunits, resulting in more efficient processing of $16 \mathrm{~S}$ rRNA. Evidently, the ability of the different ribosomal suppressor mutations to mediate efficient processing of 16S rRNA requires the presence of the RimM-YY $\rightarrow$ AA (or wild-type RimM) protein. This suggests that the poor interaction of the RimM-YY $\rightarrow$ AA protein with the $30 \mathrm{~S}$ subunits in combination with structural changes in the $30 \mathrm{~S}$ subunits brought about by the suppressor mutations can promote efficient processing of $16 \mathrm{~S}$ rRNA. Alternatively, the ribosomal suppressor mutations might partially restore the interaction of the RimM-YY $\rightarrow$ AA protein with the $30 \mathrm{~S}$ subunits, despite the fact that we have been unable to demonstrate any such effects. We have assumed that the interaction detected between RimM and 30S subunits after sucrose gradient centrifugation of cellular extracts involves immature $30 \mathrm{~S}$ subunits that contain partially processed $16 \mathrm{~S}$ rRNA, because the $\Delta$ rimM and RimM-YY $\rightarrow \mathrm{AA}$ mutants accumulate $17 \mathrm{~S}$ rRNA, and because RimM is not associated with $30 \mathrm{~S}$ subunits in $70 \mathrm{~S}$ ribosomes (containing only fully processed $16 \mathrm{~S}$ rRNA). However, the analysis of 30 S subunits copurifying with a GST-RimM protein suggests that the binding detected is with $30 \mathrm{~S}$ subunits containing fully processed $16 \mathrm{~S}$ rRNA. Possibly, two types of interactions between RimM and the 30S subunits take place: a weak interaction, which we have been unable to detect, occurs initially between RimM and the 30 S subunits that contain 17S rRNA, followed by a stronger binding, which we have observed in this study, once the $30 \mathrm{~S}$ subunits have matured further and contain fully processed 16S rRNA. Interestingly, the N84K substitution in RimM-YY $\rightarrow \mathrm{AA}$ improved processing of $16 \mathrm{~S}$ rRNA, suggesting that it did improve binding of this RimM protein to the 30 S subunits although it did not affect binding in our assay. The simplest explanation of these contradictory results, assuming that RimM is not required for the synthesis of a protein involved in $16 \mathrm{~S}$ rRNA processing, is that the N84K substitution, and possibly some of the ribosomal suppressors, such as S19H83Y, improve the proposed weak interaction between the RimM-YY $\rightarrow$ AA protein and the 30 s subunits.

\section{Are RimM and RbfA involved in 30S maturation steps both before and after processing of $16 \mathrm{~S}$ rRNA?}

RimM and RbfA bind to free 30S subunits in vivo (Dammel and Noller 1995; Bylund et al. 1997), and the GTPase Era binds to $16 \mathrm{~S}$ rRNA and $30 \mathrm{~S}$ subunits in vitro (Sayed et al. 1999). Cells lacking RimM or RbfA or cells depleted for Era accumulate 17S rRNA (Bylund et al. 1998; Inoue et al. 2003; Xia et al. 2003), and the levels of polysomes decrease with a concomitant increase in free $30 \mathrm{~S}$ and $50 \mathrm{~S}$ subunits (this paper; Dammel and Noller 1995; Inoue et al. 2003). Thus, these three proteins seem to take part in late steps of the maturation of the $30 \mathrm{~S}$ subunits that precede the conversion of $17 \mathrm{~S}$ to $16 \mathrm{~S}$ rRNA. In addition, increased expression of RbfA suppresses a cold-sensitive mutation in the $5^{\prime}$-terminal helix of $16 \mathrm{~S}$ rRNA, and RbfA was proposed to bind to this helix (Dammel and Noller 1995). The 5'-terminal helix is found only in mature 16S rRNA and not in 17S rRNA (Srivastava and Schlessinger 1989), suggesting that RbfA is involved in maturation after formation of $16 \mathrm{~S}$ rRNA. Interestingly, a mutant RbfA protein missing the 25 most carboxy-terminal amino acids does not associate stably with $30 S$ subunits and does not suppress the cold sensitivity of the helix mutation. However, the mutant RbfA protein complemented the 16S rRNA processing defect of the rbfA null mutant (Xia et al. 2003). Thus, RbfA may be involved in steps of $30 \mathrm{~S}$ maturation both before and after the conversion of $17 \mathrm{~S}$ to $16 \mathrm{~S}$ rRNA. Increased expression of RbfA partially suppresses translational defects of the $\Delta$ rim $M$ mutant (Bylund et al. 1998). Previously, we suggested that RimM participates before RbfA in 30 S maturation and that RbfA cannot bind efficiently to the $30 \mathrm{~S}$ subunits in the absence of RimM as the 30S subunits have not matured correctly. Conceivably, an increased level of RbfA would suppress a weak binding. However, in a $\Delta$ rim $M$ mutant grown at $37^{\circ} \mathrm{C}$, most RbfA molecules are associated with the $30 \mathrm{~S}$ subunits and almost no free RbfA is found (J.M. Lövgren and P.M. Wikström, unpubl.). Thus, the $30 \mathrm{~S}$ maturation deficiency of the $\Delta$ rimM mutant might be partly explained by limiting levels of free RbfA. This explanation would also account for why an increased amount of RbfA partially suppresses the defects of rimM mutants. Thus, if RimM acts before RbfA, then the reason for RbfA not being released from the $30 \mathrm{~S}$ subunits in the $\Delta$ rim $M$ mutant could be that there is a block in the next step of the maturation process. Such a step could be the binding of Era, because increased levels of Era suppress an $r b f A$ mutant (Inoue et al. 
2003). However, increased levels of Era do not suppress the slow growth of the $\Delta$ rim $M$ mutant, as would have been expected. Alternatively, RbfA might act before RimM and will normally only be released from the $30 \mathrm{~S}$ subunits when RimM has participated in the maturation process, which would explain the out-titration of RbfA in the $\Delta$ rim $M$ mutant. Consequently, RbfA and RimM might bind, in that order, to immature $30 \mathrm{~S}$ subunits and facilitate maturation steps that precede and are required for efficient conversion of $17 \mathrm{~S}$ to $16 \mathrm{~S}$ rRNA. Then, RbfA could also participate in the formation of the $5^{\prime}$ terminal helix of mature 16S rRNA before being released from the $30 \mathrm{~S}$ subunits. Possibly, RimM could be involved in maturation steps after the final processing of $16 \mathrm{~S}$ rRNA, as indicated by the presence of mature $16 \mathrm{~S}$ in the $30 \mathrm{~S}$ subunits that copurified with the GST-RimM hybrid protein.

Here we have shown that the role of RimM in ribosome maturation involves the binding to S19 in the head of the 30 S subunits. Our future strategies will aim at revealing any interplay between RimM and RbfA, and it will be interesting to determine whether RbfA takes part in maturation of the S19 region of the $30 \mathrm{~S}$ subunits.

\section{MATERIALS AND METHODS}

\section{Strains, phages, plasmids, and growth conditions}

Relevant strains, phages, and plasmids used are listed in Table 3. Strain JML068 ( $\Delta 7 \mathrm{rrn}$ rimM120/pHK-rrnC ${ }^{+}$p 70$)$ was constructed in several steps: First, pheA18::Tn10 from CAG12158 (Singer et al. 1989) was introduced into strain MW136 (rimM120) by P1 transduction selecting for $\mathrm{Tc}^{\mathrm{R}}$. From the resulting strain, MW148 (rimM120 pheA18::Tn10), the rimM120 mutation was transferred to strain TA527 $\left(\Delta 7 \mathrm{rrn} / \mathrm{pHK}-\mathrm{rrnC}^{+}\right.$pTRNA66) by selecting for the linked pheA18::Tn10, yielding strain JML042 ( $\Delta 6 r r n r r G^{+}$ rimM120 pheA18::Tn10/pHK-rrnC ${ }^{+}$pTRNA66). Then, $\Delta(r r s G-$ $g l t W$-rrlG)30::lac $Z^{+}$from strain TA527 was reintroduced by P1 transduction of strain JML042 selecting for $\mathrm{Phe}^{+}$, yielding strain JML067 ( $\Delta 7 \mathrm{rrn}$ rimM120/pHK-rrnC ${ }^{+}$pTRNA66). Finally, pTRNA66 $\left(\mathrm{Sp}^{\mathrm{R}}\right)$ of strain JML067 was replaced by the similar p70 $\left(\mathrm{Tc}^{\mathrm{R}}\right)$ plasmid from strain AVS69009 (Vila-Sanjurjo et al. 1999) by transformation, selecting for $\mathrm{Tc}^{\mathrm{R}}$ and screening for $\mathrm{Sp}^{\mathrm{S}}$. A derivative of strain JML068, JML071 ( $\Delta 7 \mathrm{rrn}$ rimM120/pKK1192U p70), was constructed by transformation of JML068 with plasmid pKK1192U $\left(\mathrm{Cb}^{\mathrm{R}}\right)$ (Makosky and Dahlberg 1987), which carries an rrnB operon with a mutation in 16S rRNA (C1192U) conferring $\mathrm{Sp}^{\mathrm{R}}$, selecting for $\mathrm{Sp}^{\mathrm{R}}$ and $\mathrm{Cb}^{\mathrm{R}}$, and screening for $\mathrm{Km}^{\mathrm{S}}$ (i.e., the loss of pHK-rrnC ${ }^{+}$). Strains JML113 and JML114 containing $\Delta$ rimM102 instead of rimM120 were constructed in a similar way as JML068 and JML071, respectively.

Plasmid pJML005 that carries a fusion between the glutathione S-transferase gene ( $g s t)$ and rimM (codons 2-182) was constructed by PCR-amplifying the wild-type rim $M$ with oligonucleotides $5^{\prime}$ TTTTGGATCCAAACAACTCACCGCGCAAG- $3^{\prime}$ and $5^{\prime}$-TTTT GAATTCTTAAAAACCAGGGTCCCAATC-3', digesting the PCR fragment with BamHI and EcoRI, and inserting it into similarly digested pGEX-4T-2. The latter oligonucleotide destroyed the BamHI site in the $3^{\prime}$-end of rimM without changing the encoded amino acid sequence. Plasmid pJML051 expressing a GST$\operatorname{RimM}(\mathrm{YY} \rightarrow \mathrm{AA})$ hybrid protein was constructed in the same way using chromosomal DNA from the RimM-YY $\rightarrow$ AA mutant MW136 as the template for PCR amplification.

Rich medium used was LB (Bertani 1951). Cultures were grown at indicated temperatures, and the growth was monitored at 600 nm using a Shimadzu UV-1601 spectrophotometer.

\section{Isolation of rimM mutants}

Random mutagenesis of rimM was done using hydroxylamine. Ten $\mu \mathrm{g}$ of plasmid pMW279, which carries the rimM gene, was mutagenized at $75^{\circ} \mathrm{C}$ with $1 \mathrm{M}$ of hydroxylamine for $90 \mathrm{~min}$, and then dialyzed against water. The wild-type strain MW100 was transformed with the mutagenized pMW279, and transformants were transduced to kanamycin resistance using $\lambda 439 \Delta$ rimM-2 (Persson et al. 1995). Two rimM alleles complemented the slow growth at $37^{\circ} \mathrm{C}$ but not at $42^{\circ} \mathrm{C}$; however, they contained two mutations each. One of these alleles, in plasmid pMW316, encoded a RimM protein with two amino acid substitutions, G17E and G121D, while the other allele, in plasmid pMW317, encoded a RimM protein with a single substitution, G27R, and contained a silent mutation in codon 13. The two mutations in pMW316 were cloned apart, yielding plasmids pMW324 (RimM-G17E) and pMW334 (RimM-G121D). The silent mutation in pMW317 was removed by PCR, yielding plasmid pMW325 (RimM-G27R).

Oligonucleotide-directed mutagenesis by using either singlestranded M13 DNA templates (Kunkel 1985; Kunkel et al. 1987) or overlap extension PCR (Ho et al. 1989) was done to create specific amino acid substitutions in RimM. The mutations were cloned into plasmid pMW296 carrying a 4.6-kb chromosomal fragment that contains the rimM region. This fragment was then transferred to plasmid pMAK705, which was used to introduce the mutant alleles into the E. coli chromosome as described (Hamilton et al. 1989).

\section{Isolation of suppressor mutations}

To isolate faster-growing derivatives of the RimM-YY $\rightarrow \mathrm{AA}$ mutants MW136 and JML068, several cultures were inoculated from independent colonies and grown overnight in rich liquid medium. An aliquot of each overnight culture was streaked out on rich medium plates and subinoculated into fresh liquid medium. This procedure was repeated until revertants appeared on the plates. Only one revertant from each original culture was saved for further analyses. To investigate whether the suppressor mutations in strain JML068 were plasmid-carried, plasmid DNA from the suppressor mutants was used to transform strain JML071 selecting for $\mathrm{Km}^{\mathrm{R}}$ (pHK-rrnC) and screening for $\mathrm{Sp}^{\mathrm{s}}$. Plasmid preparations that conferred fast growth were used to transform strain $\mathrm{DH} 5 \alpha$ (Hanahan 1983) selecting for $\mathrm{Km}^{\mathrm{R}}$ and screening for $\mathrm{Tc}^{\mathrm{S}}$, to obtain clones that only contained the $\mathrm{pHK}$-rrnC plasmid and not the p70 tRNA plasmid. Plasmid DNA from such $\mathrm{Km}^{\mathrm{R}} \mathrm{Tc}^{\mathrm{S}} \mathrm{DH} 5 \alpha$ derivatives was used to transform strain JML071, selecting for $\mathrm{Km}^{\mathrm{R}}$, to investigate whether the suppressor mutations were on the rrnplasmid. 
RimM binds to $\mathrm{S19}$ in the 305 subunits

TABLE 3. Bacterial strains, bacteriophages, and plasmids

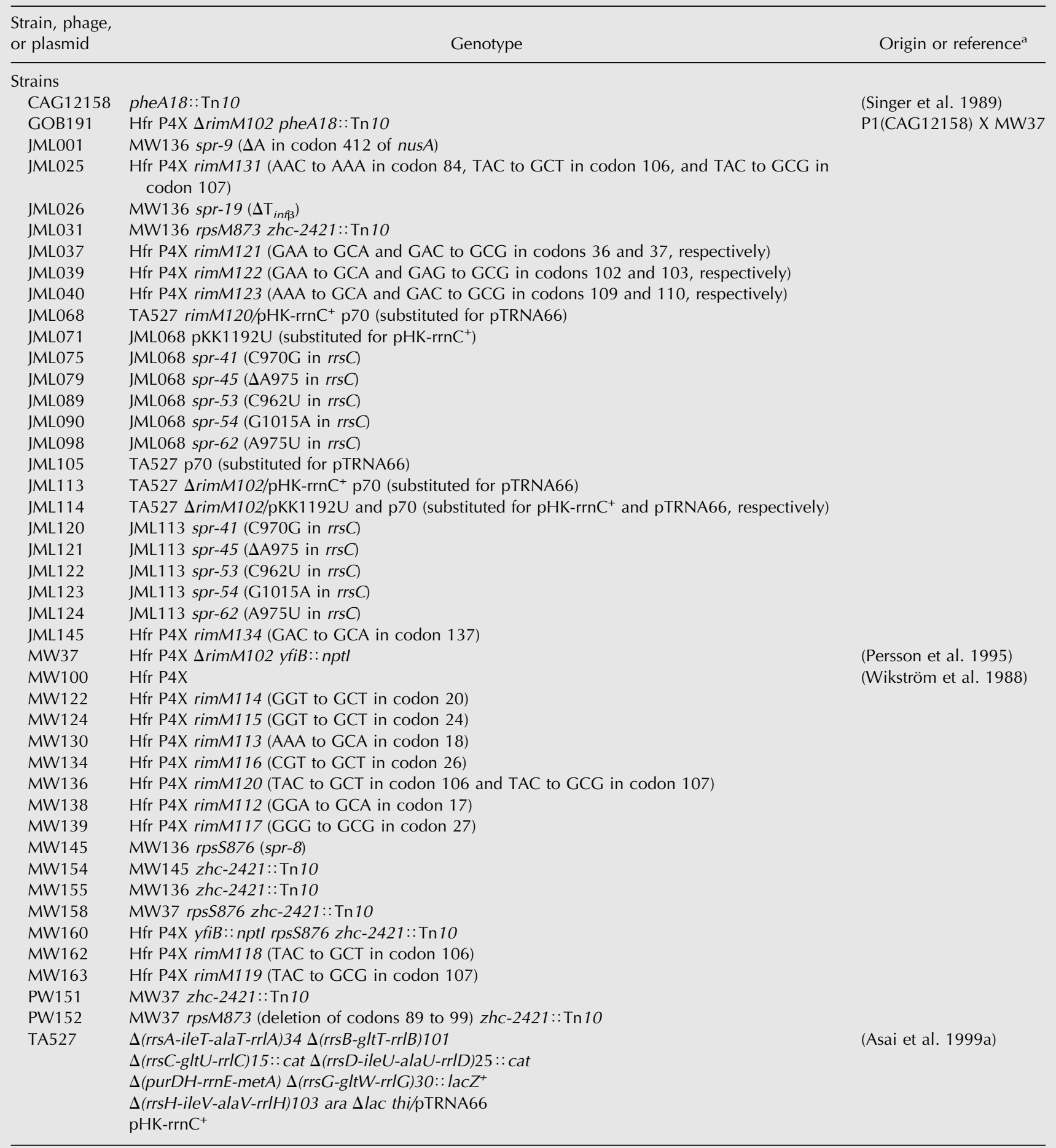

(continued)

\section{Ribosome profiles after sucrose gradient centrifugation}

Cell cultures were grown in $\mathrm{LB}$ at $37^{\circ} \mathrm{C}$ to 70 Klett units. Polysomes were stabilized by addition of chloramphenicol as described (Brow and Noller 1983). Cells were harvested by centrifugation, and polysome extracts were prepared by a previously described freeze-thaw and lysozyme method (Ron et al. 1966) and fractionated by sucrose gradient centrifugation according to Powers and Noller (1990), with the exception that the sucrose was prepared in $10 \mathrm{mM}$ Tris- $\mathrm{HCl}, \mathrm{pH}$ 7.5, $50 \mathrm{mM} \mathrm{KCl,} 6 \mathrm{mM} \beta$-mercaptoethanol, $10 \mathrm{MgCl}_{2}$. For ribosome profiles under conditions that dissociate 
TABLE 3. Continued

\begin{tabular}{|c|c|c|}
\hline $\begin{array}{l}\text { Strain, phage, } \\
\text { or plasmid }\end{array}$ & Genotype & Origin or reference ${ }^{a}$ \\
\hline \multicolumn{3}{|l|}{ Bacteriophages } \\
\hline 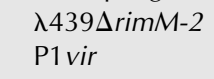 & srimM102 yfiB:: nptl & $\begin{array}{l}\text { (Persson et al. 1995) } \\
\text { Laboratory stock }\end{array}$ \\
\hline \multicolumn{3}{|l|}{ Plasmids } \\
\hline pGEX-4T-2 & bla lacla $\mathrm{P}_{\text {tac }}-g s t$ & Amersham Biosciences \\
\hline pJML005 & bla lacla $\mathrm{P}_{\text {tac }}{ }^{-g s t-r i m M^{+}}$ & \\
\hline pJML051 & bla lacl ${ }^{1} \mathrm{P}_{\text {tac }}$-gst-rimM120 & \\
\hline pMAK705 & cat & (Hamilton et al. 1989) \\
\hline pMW279 & $P_{\text {lac }}$-rimM $M^{+}$-cat-rrnBT $T_{1}$ bla & (Persson et al. 1995) \\
\hline pMW296 & $\mathrm{ffh}^{+} r p s P^{+}-\mathrm{rimM} \mathrm{M}^{+}$-trmD ${ }^{+}-r p / S^{+}$yfiB ${ }^{+}$bla & \\
\hline pMW316 & pMW279 rimM107 (GGA to GAA in codon 17 and GGC to GAC in codon 121) & \\
\hline pMW317 & pMW279 rimM108 (CCC to CCT in codon 13 and GGG to AGG in codon 27) & \\
\hline pMW324 & pMW279 rimM109 (GGA to GAA in codon 17) & \\
\hline pMW325 & pMW279 rimM111 (GGG to AGG in codon 27) & \\
\hline pMW334 & pMW279 rimM110 (GGC to GAC in codon 121) & \\
\hline
\end{tabular}

annless otherwise noted, the origin was this study.

the ribosomal subunits, S30 extracts were prepared and fractionated by sucrose gradient centrifugation as previously described (Bylund et al. 1997).

\section{Primer extension on rRNA}

Total RNA was prepared using the Qiagen RNA/DNA and Qiagen RNeasy Kits (QIAGEN). RNA from ribosomal particles purified by sucrose gradient centrifugation was prepared by three successive extractions with phenol/chloroform/isoamyl alcohol (25:24:1). The RNA was then precipitated by ethanol and dissolved in $50 \mu \mathrm{L}$ of water. Primer extension was performed as described earlier (Bylund et al. 1998).

\section{Preparation of cell extracts and purification of GST-RimM proteins}

One-hundred-mL cultures were grown in LB medium to 70 Klett Units, harvested by centrifugation, and resuspended in $0.5 \mathrm{~mL}$ of $20 \mathrm{mM}$ Tris- $\mathrm{HCl} \mathrm{pH} \mathrm{7.0,} 150 \mathrm{mM} \mathrm{NaCl}$. Cells were disrupted either by $10-15$ cycles of 5 -sec sonication at setting $20 \%$ on a Vibra cell VCX400 (Sonics and Materials), or by freeze-thawing three times in the presence of lysozyme $(1 \mu \mathrm{g} / \mathrm{mL})$. RNase-free DNase I $(10 \mathrm{U})$ was added, cell debris was removed by centrifugation, and the cell extracts were adsorbed to $0.1 \mathrm{~mL}$ of Glutathione Sepharose 4B (Amersham Biosciences). The Sepharose was packed into MicroSpin columns (Amersham Biosciences) and washed with $0.5 \mathrm{~mL}$ volumes of $20 \mathrm{mM}$ Tris- $\mathrm{HCl}, \mathrm{pH} 7.0$, containing increasing concentrations of $\mathrm{NaCl}$. Finally, the GST-RimM fusions were eluted from the columns with $0.5 \mathrm{~mL}$ of $10 \mathrm{mM}$ reduced glutathione (Sigma-Aldrich) in $50 \mathrm{mM}$ Tris- $\mathrm{HCl}, \mathrm{pH}$ 8.0.

\section{Mass spectrometric analyses}

Total protein in fractions from purification of GST-RimM proteins was precipitated with acetone, dissolved, digested with trypsin according to the manufacturer's directions (Trypsin Gold; Promega), and purified on a ZipTip (Millipore). Peptide separation and ESI-MS/MS (Q-TOF Ultima Waters-Micromass, MS Technologies) were performed as described (Szkanderova et al. 2003) by using a reverse-phase C18 column (Atlantis, NanoEase $75 \mu \mathrm{m} \times 150 \mathrm{~mm}$ ) and analyzed with ProteinLynx GlobalSERVER version 2.05 (Waters-Micromass, MS Technologies). Peptides were identified using the Swiss-Prot database (Swiss Institute of Bioinformatics, Geneva, Switzerland).

Protein bands were cut out from colloidal Coomassie-stained (Neuhoff et al. 1990) polyacrylamide gels and digested according to Shevchenko et al. (1996). The extracted peptide solution was purified on a ZipTip and analyzed by MALDI mass spectrometry (Voyager-DE STR, Applied Biosystems) according to Szkanderova et al. (2003). Proteins were identified by using the SwissProt database and the Mascot search engine (www.matrixscience. com).

\section{ACKNOWLEDGMENTS}

We thank Dr. Catherine L. Squires for kindly providing strain TA527, Dr. Antón Vila-Sanjurjo for kindly providing strains AVS69009 and AVS69009/pKK1192U, and Dr. Debra Milton for helpful comments on the manuscript. O.P.P. was supported by the Swedish Natural Science Research Council (B-BU 10664). P.M.W. was supported by the Swedish Natural Science Research Council (B-BU 9911), the Swedish Research Council (621-2001-2171), the Magnus Bergvall Foundation, and the Kempe Foundations. O.P.P. and P.M.W. were jointly supported by the Carl Trygger Foundation. G.W. was supported by the Kempe Foundations and the Swedish Research Council for Environment, Agricultural Sciences and Spatial Planning.

Received April 28, 2004; accepted July 26, 2004. 


\section{REFERENCES}

Alix, J.-H. and Guérin, M.-F. 1993. Mutant DnaK chaperones cause ribosome assembly defects in Escherichia coli. Proc. Natl. Acad. Sci. 90: 9725-9729.

Anantharaman, V. and Aravind, L. 2002. The PRC-barrel: A widespread, conserved domain shared by photosynthetic reaction center subunits and proteins of RNA metabolism. Genome Biol. 3: research0061.0061-research0061.0069.

Asai, T., Condon, C., Voulgaris, J., Zaporojets, D., Shen, B., Al-Omar, M., Squires, C., and Squires, C.L. 1999a. Construction and initial characterization of Escherichia coli strains with few or no intact chromosomal rRNA operons. J. Bacteriol. 181: 3803-3809.

Asai, T., Zaporojets, D., Squires, C., and Squires, C.L. 1999b. An Escherichia coli strain with all chromosomal rRNA operons inactivated: Complete exchange of rRNA genes between bacteria. Proc. Natl. Acad. Sci. 96: 1971-1976.

Bertani, G. 1951. Studies on lysogenesis. I. The mode of phage liberation by lysogenic Escherichia coli. J. Bacteriol. 62: 293-300.

Brow, D.A. and Noller, H.F. 1983. Protection of ribosomal RNA from kethoxal in polyribosomes. Implication of specific sites in ribosome function. J. Mol. Biol. 163: 27-46.

Bylund, G.O., Persson, B.C., Lundberg, L.A.C., and Wikström, P.M. 1997. A novel ribosome-associated protein is important for efficient translation in Escherichia coli. J. Bacteriol. 179: 4567-4574.

Bylund, G.O., Wipemo, L.C., Lundberg, L.A.C., and Wikström, P.M. 1998. RimM and RbfA are essential for efficient processing of $16 \mathrm{~S}$ rRNA in Escherichia coli. J. Bacteriol. 180: 73-82.

Bylund, G.O., Lövgren, J.M., and Wikström, P.M. 2001. Characterization of mutations in the metY-nusA-infB operon that suppress the slow growth of a $\Delta$ rimM mutant. J. Bacteriol. 183: 60956106.

Charollais, J., Pflieger, D., Vinh, J., Dreyfus, M., and Iost, I. 2003. The DEAD-box RNA helicase SrmB is involved in the assembly of $50 \mathrm{~S}$ ribosomal subunits in Escherichia coli. Mol. Microbiol. 48: 12531265.

Charollais, J., Dreyfus, M., and Iost, I. 2004. CsdA, a cold-shock RNA helicase from Escherichia coli, is involved in the biogenesis of $50 \mathrm{~S}$ ribosomal subunit. Nucleic Acids Res. 32: 2751-2759.

Dammel, C.S. and Noller, H.F. 1995. Suppression of a cold-sensitive mutation in 16S rRNA by overexpression of a novel ribosomebinding factor, RbfA. Genes \& Dev. 9: 626-637.

El Hage, A., Sbaï, M., and Alix, J.H. 2001. The chaperonin GroEL and other heat-shock proteins, besides DnaK, participate in ribosome biogenesis in Escherichia coli. Mol. Gen. Genet. 264: 796-808.

Fuller-Pace, F.V. 1994. RNA helicases: Modulators of RNA structure. Trends Cell Biol. 4: 271-274.

Fuller-Pace, F.V., Nicol, S.M., Reid, A.D., and Lane, D.P. 1993. DbpA: A DEAD box protein specifically activated by $23 \mathrm{~S}$ rRNA. EMBO J. 12: 3619-3626.

Gibson, T.J., Thompson, J.D., and Heringa, J. 1993. The KH domain occurs in a diverse set of RNA-binding proteins that include the antiterminator NusA and is probably involved in binding to nucleic acid. FEBS Lett. 324: 361-366.

Hamilton, C.M., Aldea, M., Washburn, B.K., Babitzke, P., and Kushner, S.R. 1989. New method for generating deletions and gene replacements in Escherichia coli. J. Bacteriol. 171: 4617-4622.

Hanahan, D. 1983. Studies on transformation of Escherichia coli with plasmids. J. Mol. Biol. 166: 557-580.

Ho, S.N., Hunt, H.D., Horton, R.M., Pullen, J.K., and Pease, L.R. 1989. Site-directed mutagenesis by overlap extension using the polymerase chain reaction. Gene 77: 51-59.

Holmes, K.L. and Culver, G.M. 2004. Mapping structural differences between 30 S ribosomal subunit assembly intermediates. Nat. Struct Mol. Biol. 11: 179-186.

Inoue, K., Alsina, J., Chen, J., and Inouye, M. 2003. Suppression of defective ribosome assembly in a $r b f A$ deletion mutant by overexpression of Era, an essential GTPase in Escherichia coli. Mol. Microbiol 48: 1005-1016.
Jones, P.G. and Inouye, M. 1996. RbfA, a 30 S ribosomal binding factor, is a cold-shock protein whose absence triggers the coldshock response. Mol. Microbiol. 21: 1207-1218.

Kaczanowska, M. and Rydén-Aulin, M. 2004. Temperature sensitivity caused by mutant release factor 1 is suppressed by mutations that affect 16S rRNA maturation. J. Bacteriol. 186: 30463055.

Kunkel, T.A. 1985. Rapid and efficient site-specific mutagenesis without phenotypic selection. Proc. Natl. Acad. Sci. 82: 488-492.

Kunkel, T.A., Roberts, J.D., and Zakour, R.A. 1987. Rapid and efficient site-specific mutagenesis without phenotypic selection. Methods Enzymol. 154: 367-382.

Li, Z., Pandit, S., and Deutscher, M.P. 1999. RNase G (CafA protein) and RNase $\mathrm{E}$ are both required for the $5^{\prime}$ maturation of $16 \mathrm{~S}$ ribosomal RNA. EMBO J. 18: 2878-2885.

Liu, M.Y., Yang, H., and Romeo, T. 1995. The product of the pleiotropic Escherichia coli gene csrA modulates glycogen biosynthesis via effects on mRNA stability. J. Bacteriol. 177: 2663-2672.

Lövgren, J.M. and Wikström, P.M. 2001. Hybrid protein between ribosomal protein S16 and RimM of Escherichia coli retains the ribosome maturation function of both proteins. J. Bacteriol. 183: 5352-5357.

Maki, J.A., Southworth, D.R., and Culver, G.M. 2003. Demonstration of the role of the DnaK chaperone system in assembly of $30 \mathrm{~S}$ ribosomal subunits using a purified in vitro system. RNA 9: 14181421.

Makosky, P.C. and Dahlberg, A.E. 1987. Spectinomycin resistance at site 1192 in $16 \mathrm{~S}$ ribosomal RNA of E. coli: An analysis of three mutants. Biochimie 69: 885-889.

Nashimoto, H. 1993. Non-ribosomal proteins affecting the assembly of ribosomes in Escherichia coli. In The translational apparatus (eds. K.H. Nierhaus et al.), pp. 185-195. Plenum Press, New York.

Nashimoto, H., Miura, A., Saito, H., and Uchida, H. 1985. Suppressors of temperature-sensitive mutations in a ribosomal protein gene, rpsL (S12), of Escherichia coli K12. Mol. Gen. Genet. 199: 381387.

Neuhoff, V., Stamm, R., Pardowitz, I., Arold, N., Ehrhardt, W., and Taube, D. 1990. Essential problems in quantification of proteins following colloidal staining with coomassie brilliant blue dyes in polyacrylamide gels, and their solution. Electrophoresis 11: 101117.

Nierhaus, K.H. 1991. The assembly of prokaryotic ribosomes. Biochimie 73: 739-755.

Nishi, K., Morel-Deville, F., Hershey, J.W.B., Leighton, T., and Schnier, J. 1988. An eIF-4A-like protein is a suppressor of an Escherichia coli mutant defective in 50 S ribosomal subunit assembly. Nature 336: 496-498.

Persson, B.C., Bylund, G.O., Berg, D.E., and Wikström, P.M. 1995. Functional analysis of the ffh-trmD region of the Escherichia coli chromosome by using reverse genetics. J. Bacteriol. 177: 55545560.

Powers, T. and Noller, H.F. 1990. Dominant lethal mutations in a conserved loop in 16S rRNA. Proc. Natl. Acad. Sci. 87: 10421046.

Ron, E.Z., Kohler, R.E., and Davis, B.D. 1966. Polysomes extracted from Escherichia coli by freeze-thaw-lysozyme lysis. Science 153: $1119-1120$.

Sayed, A., Matsuyama, S., and Inouye, M. 1999. Era, an essential Escherichia coli small G-protein, binds to the $30 \mathrm{~S}$ ribosomal subunit. Biochem. Biophys. Res. Comm. 264: 51-54.

Shevchenko, A., Wilm, M., Vorm, O., and Mann, M. 1996. Mass spectrometric sequencing of proteins silver-stained polyacrylamide gels. Anal. Chem. 68: 850-858.

Singer, M., Baker, T.A., Schnitzler, G., Deischel, S.M., Goel, M., Dove, W., Jaacks, K.J., Grossman, A.D., Erickson, J.W., and Gross, C.A. 1989. A collection of strains containing genetically linked alternating antibiotic resistance elements for genetic mapping of Escherichia coli. Microbiol. Rev. 53: 1-24. 


\section{Lövgren et al.}

Srivastava, A.K. and Schlessinger, D. 1989. Processing pathway of Escherichia coli 16S precursor rRNA. Nucleic Acids Res. 17: 16491663.

Szkanderova, S., Hernychova, L., Kasalova, I., Vavrova, J., Stulik, J., Abend, M., and van Beuningen, D. 2003. Proteomic analysis of radiation-induced alterations in L929 cells. Folia Biol. (Praha) 49: $15-25$.

Toone, W.M., Rudd, K.E., and Friesen, J.D. 1991. deaD, a new Escherichia coli gene encoding a presumed ATP-dependent RNA helicase, can suppress a mutation in $r p s B$, the gene encoding ribosomal protein S2. J. Bacteriol. 173: 3291-3302.

Tsu, C.A. and Uhlenbeck, O.C. 1998. Kinetic analysis of the RNAdependent adenosinetriphosphatase activity of DbpA, an Escherichia coli DEAD protein specific for $23 \mathrm{~S}$ ribosomal RNA. Biochemistry 37: 16989-16996.

Vila-Sanjurjo, A., Squires, C.L., and Dahlberg, A.E. 1999. Isolation of kasugamycin resistant mutants in the $16 \mathrm{~S}$ ribosomal RNA of Escherichia coli. J. Mol. Biol. 293: 1-8.

Wikström, P.M., Byström, A.S., and Björk, G.R. 1988. Non-autogenous control of ribosomal protein synthesis from the trmD operon in Escherichia coli. J. Mol. Biol. 203: 141-152.

Wimberly, B.T., Brodersen, D.E., Clemons, W.M., Morgan-Warren, R.J., Carter, A.P., Vonrhein, C., Hartsch, T., and Ramakrishnan, V. 2000. Structure of the 30 S ribosomal subunit. Nature 407: 327 339.

Xia, B., Ke, H., Shinde, U., and Inouye, M. 2003. The role of RbfA in $16 \mathrm{~S}$ rRNA processing and cell growth at low temperature in Escherichia coli. J. Mol. Biol. 332: 575-584.

Young, R.A. and Steitz, J.A. 1978. Complementary sequences 1700 nucleotides apart form a ribonuclease III cleavage site in Escherichia coli ribosomal precursor RNA. Proc. Natl. Acad. Sci. 75: 3593-3597. 

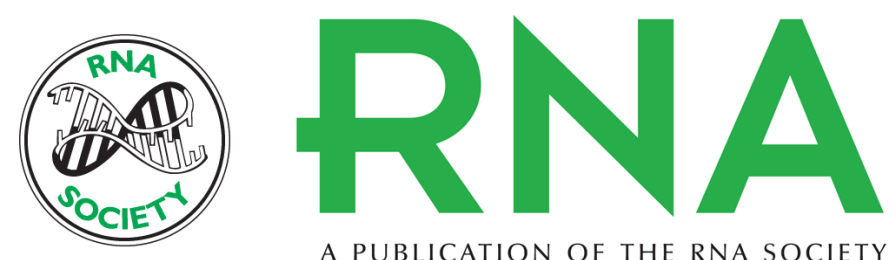

A PUBLICATION OF THE RNA SOCIETY

\section{The PRC-barrel domain of the ribosome maturation protein RimM mediates binding to ribosomal protein $\mathrm{S} 19$ in the $30 \mathrm{~S}$ ribosomal subunits}

J. MATTIAS LÖVGREN, GÖRAN O. BYLUND, MANOJ K. SRIVASTAVA, et al.

RNA 2004 10: 1798-1812

References This article cites 48 articles, 21 of which can be accessed free at:

http://rnajournal.cshlp.org/content/10/11/1798.full.html\#ref-list-1

License

Email Alerting Receive free email alerts when new articles cite this article - sign up in the box at the

Service top right corner of the article or click here. 